\title{
High-resolution state-selected ion-molecule reaction studies using pulsed field ionization photoelectron-secondary ion coincidence method
}

\author{
X.-M. Qian, T. Zhang, C. Chang, P. Wang, and C. Y. Ng ${ }^{\text {a) }}$ \\ Department of Chemistry, University of California at Davis, One Shields Avenue, Davis, California 95616 \\ Yu-Hui Chiu and Dale J. Levandier \\ Institute for Scientific Research, Boston College, Newton, Massachusetts 02159 \\ J. Scott Miller ${ }^{\text {b) }}$ and Rainer A. Dressler ${ }^{\mathrm{c}}$ \\ Air Force Research Laboratory, Space Vehicles Directorate, Hanscom AFB, Massachusetts 01731-3010 \\ Tomas Baer \\ Department of Chemistry, The University of North Carolina, Chapel Hill, North Carolina 27599-3290
}

Darcy S. Peterka

Chemical Science Division, Lawrence Berkeley National Laboratory, Berkeley, California 94720

(Received 21 March 2003; accepted 6 June 2003)

\begin{abstract}
We have developed an octopole-quadrupole photoionization apparatus at the Advanced Light Source for absolute integral cross-section measurements of rovibrational-state-selected ion-molecule reactions. This apparatus consists of a high-resolution photoionization ion source, a wired ion gate lens, a dual radio-frequency (rf) octopole ion guide reaction gas cell, and a quadrupole mass spectrometer for reactant and product ion detection. The unique feature of this apparatus is the implementation of the high-resolution pulsed field ionization-photoelectron (PFI-PE)-photoion coincidence (PFI-PEPICO) technique, which has allowed the rotational-state selection of diatomic ions for ion-molecule reaction studies. The novel application of the wired ion gate lens for the rejection of false coincidence background ions is described. This application, along with the differential-ion-gate scheme, has made possible the measurements of rovibrational-state-selected absolute integral reaction cross sections for ion-molecule collisions using the PFI-PE-secondary ion coincidence (PFI-PESICO) method. The successful measurement of absolute state-selected cross sections for $\mathrm{H}_{2}{ }^{+}\left(X, v^{+}, N^{+}\right)+\mathrm{Ar}(\mathrm{Ne})$ with $v^{+}$up to 17 [the third to the last vibrational state of $\left.\mathrm{H}_{2}{ }^{+}(X)\right]$ demonstrates the high sensitivity of this differential-ion-gate PFI-PESICO method. In order to gain a detailed understanding and to obtain optimal performance of the wired ion gate lens for PFI-PESICO measurements, we have carried out ion trajectory calculations of reactant ions between the photoionization region and the rf-octopole ion guide. On the basis of these calculations, possible future improvements for the application of this differential-ion-gate PFI-PESICO scheme are discussed. (C) 2003 American Institute of Physics.
\end{abstract}

[DOI: $10.1063 / 1.1599071]$

\section{INTRODUCTION}

Photoionization is the most versatile method for the preparation of state- or energy-selected reactant ions. ${ }^{1-7}$ Single-photon ionization, which usually occurs in the vacuum ultraviolet (VUV), is among the most general and cleanest photoionization schemes. ${ }^{1-6}$ Because of the fine control in photon energy, simple reactant ions in their ground states with $100 \%$ purity or in a well-defined distribution of internal states can be easily prepared by photoionization. ${ }^{2-4}$ By virtue of the photoionization mechanism, excited reactant ions in metastable states, which cannot be produced by photoexcitation of ions, can also be formed by VUV photoionization. ${ }^{1-4}$

\footnotetext{
a)Electronic mail: cyng@chem.ucdavis.edu

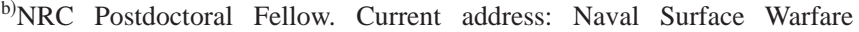
Center-Indian Head, 101 Strauss Avenue, Indian Head, MD 20640.

${ }^{c}$ Electronic mail: Rainer.Dressler@hanscom.af.mil
}

The threshold photoelectron (TPE)-photoion coincidence (TPEPICO) method, ${ }^{5,6}$ which concerns the detection of correlated TPE-photoion pairs using tunable VUV radiation, represents a more advanced photoionization technique for the preparation of state- or energy-selected ions. In such experiments, the internal state or energy of photoions detected in coincidence of TPEs is equal to the difference between the photoionization energy and the IE of the neutral precursor because the TPEs have near-zero kinetic energies. Due to the mediation of near-resonant autoionizing states, ${ }^{8}$ the TPE bands for vibronic states of photoions with negligible Franck-Condon factors for direct photoionization can be observed. Thus, reactant ions in a wide range of internal states or energies can, in principle, be prepared using the TPEPICO method. If the product ions formed by ion-neutral collisions involving the state-selected reactant ions maintain the time correlation with the TPE triggering signals, the reactivity of the state- or energy-selected reactant ions can be examined 
by coincidence detection of the TPE-product ion pairs. This technique, which is known in the literature as the TPEsecondary ion coincidence (TPESICO) method, ${ }^{5}$ has been demonstrated to be a general scheme for state-selected ionmolecule reaction studies. However, previous applications ${ }^{5,6}$ of the TPESICO method for state-selected ion-molecule collisions experiments have been constrained by the relatively poor TPE resolution $[\geqslant 20 \mathrm{meV}$, full width at half maximum (FWHM)] and experimental sensitivity, limiting reactivity studies to reactant ions prepared in relatively low vibrational levels. The problem associated with the hot-electron tail in TPE detection ${ }^{8}$ can also degrade the purity of vibronic states prepared in TPESICO experiments. The use of the penetrating field scheme, along with the time-of-flight (TOF) discrimination of hot electrons in TPE measurements has shown to greatly reduce the hot-tail problem. ${ }^{9,10}$ The successful application of the penetration field and TOF method in synchrotron based TPEPICO measurements of rare gas dimers has achieved TPE resolutions of $2-3 \mathrm{meV}$ (FWHM) ${ }^{10}$ However, this TOF discrimination method requires a single-bunch or a two-bunch synchrotron operation, a mode that has a significantly lower light intensity than that of a multibunch synchrotron operation.

The pulsed field ionization (PFI)-photoelectron (PFI-PE) scheme $^{11-16}$ is a variant of the TPE method and has been shown to be free from the hot-tail problem. The highresolution PFI-PE vibrational bands for many diatomic and polyatomic hydride species have been recorded with rotational resolution even for vibrational states close to the ion dissociation limits. ${ }^{14-16}$ We have recently developed a generally applicable synchrotron based PFI-PE-photoion coincidence (PFI-PEPICO) scheme, making it possible to select the internal state or energy of reactant ions with a resolution of $\approx 5-10 \mathrm{~cm}^{-1}$ (FWHM) for unimolecular dissociation studies. ${ }^{14,16,17}$ Since laser-based PFI-PE studies are, typically, conducted at a repetition rate of $<100 \mathrm{~Hz}$, it is not suitable to employ the coincidence technique for the state selection of reactant ions. The state selection of reactant ions can be made by the PFI-photoion (PFI-PI). ${ }^{13}$ We note that both the PFI-PEPICO and laser based PFI-PI methods are concerned with the selection of PFI-PIs in well-defined internal states. In the laser based PFI-PI method, the PFI-PI detection is timed with respect to the excitation laser pulse, whereas the PFI-PI detection in the case of PFI-PEPICO measurements is timed with respect to the PFI-PE pulses. The PFI-PI scheme ${ }^{13}$ usually requires a relatively large dc field and/or a relatively long delay between the VUV excitation and PFI field in order to separate background prompt ions from PFIPIs. The higher dc field used has the effect of inducing field ionization of high- $n$ Rydberg species initially populated by VUV excitation, and thus lowers the sensitivity and resolution. The PFI-PEPICO method has the potential to be a more sensitive and a higher resolution method than the PFI-PI scheme because the use of a high dc field is not required. However, a major limitation in the coincidence approach is the background noise resulting from false coincidences. This is especially problematic in high-resolution studies where the true energy selected ions consist of a very small fraction of all the ions that are produced.
As shown in a previous analysis, ${ }^{16}$ the signal-to-noise $(\mathrm{S} / \mathrm{N})$ for PFI-PEPICO (TPEPICO) studies of unimolecular reactions is calculated as

$$
\frac{S}{N}=\sqrt{\frac{N_{t} f_{e} f_{i} t}{1+2 N \Delta t}},
$$

whereas that for PFI-PE-secondary ion coincidence (PFIPESICO) or TPESICO studies ${ }^{16}$ of bimolecular ion-molecule collisions is predicted to be

$$
\frac{S}{N}=\sqrt{\frac{N_{t} f_{e} f_{i} n l \sigma t}{1+2 N \Delta t^{\prime}}} .
$$

Here, $N_{t}, N, f_{e}, f_{i}$, and $t$ represent the threshold ionization rate, the total ionization rate, the electron transmission, the ion transmission, and the data accumulation time, respectively. For PFI-PEPICO TOF measurements, the full width $(\Delta t)$ for the TOF peak of a daughter ion is, typically, equal to a few $\mu$ s. In reactions that involve the formation of slow product ion, such as in charge transfer reactions, the full width $\left(\Delta t^{\prime}\right)$ for slow product ions can span the TOF range of $\approx 100-200 \mu \mathrm{s}$. Taking into account that typical total cross sections $\left(\sigma \approx 10^{-16}-10^{-18} \mathrm{~cm}^{2}\right)$ for ion-neutral collisions and the typical density $\left(n \approx 2 \times 10^{-4}\right.$ Torr or 6 $\times 10^{12}$ molecules $/ \mathrm{cm}^{3}$ ) for neutral reactants ${ }^{2-4}$ and the length $(l \approx 5 \mathrm{~cm})$ for the reaction gas cell used, we expect that the S/N for PFI-PESICO (TPESICO) TOF measurements of bimolecular reactions is generally more than two orders of magnitude lower than that for PFI-PEPICO (TPEPICO) TOF measurements of unimolecular dissociation reactions. Thus, the success of the PFI-PESICO scheme depends critically on the efficient rejection of false coincidence background ions.

In this article, we report the development of the PFI-PEsecondary ion coincidence (PFI-PESICO) method for stateselected ion-neutral collision studies at the Chemical Dynamics Beamline ${ }^{18}$ of the Advanced Light Source (ALS). We have achieved the optimal rejection of false coincidence ions by the novel application of a wired ion gate lens, which is referred to as an ion gate below. ${ }^{19,20}$ This gate can be opened for a very short period $(>20 \mathrm{~ns})$ to let the true ions pass while rejecting the false ions during its closed period. This scheme of using an ion gate to pass only the true coincidence ions to the ion detector as signified by the threshold electron signals has been employed in previous TPEPICO studies. ${ }^{21,22}$ Due to the random nature of false coincidence ions, some false coincidence ions are expected to pass to the ion detector together with the true coincidence ions when the ion gate is on. In order to correct for this false coincidence background, a second ion gate with the same gate width is opened at an arbitrary delay with respect to the first ion gate, which only samples the false coincidence ions. Since the false coincidence ions are produced randomly and have a uniform intensity in time, the true coincidence ion intensity can be obtained by taking the difference between the ion counts associated with the first and second ion gates. This differential-ion-gate coincidence scheme has been successfully used previously for measuring the TPE spectra of van der Waals dimers formed by supersonic expansions and radi- 
cals produced in gaseous discharge despite the fact that the ion-gate width used was significantly greater than those employed in the present study. ${ }^{21,22}$ As shown below, the narrow ion-gate width achieved using the wired ion gate lens, together with the application of the differential-ion-gate coincidence scheme, is critical for the success of the present PFIPESICO experiment. The procedures for fabrication of the wired ion gate lens are described here. In order to gain a detailed understanding concerning the performance of the ion gate, we have also performed trajectory calculations of photoions between the photoionization region and the rfoctopole ion guide. These calculations have not only provided guidance in setting the parameters for the optimal operation of the ion gate, but have also yielded useful insight on possible improvements of the PFI-PESICO scheme.

Experimental cross sections on the state-selected ionmolecule reactions $\operatorname{Ar}^{+}\left({ }^{2} \mathrm{P}_{3 / 2,1 / 2}\right)+\mathrm{D}_{2}$ and $\mathrm{H}_{2}{ }^{+}\left(v^{+}\right.$ $\left.=0-17, N^{+}=1\right)+\operatorname{Ar}(\mathrm{Ne})$ have been obtained using this PFI-PESICO scheme. ${ }^{23}$ Selected results are presented here to illustrate the application of the differential-pulse-ion-gate scheme for PFI-PEPICO and PFI-PESICO measurements. With further improvement in the kinetic energy resolution for reactant ions, we believe that this new PFI-PESICO scheme will play an important role in providing accurate new state-selected cross-section data for simulation of ionmolecule reaction cycles in plasma and planetary atmospheres, in addition to providing new challenges for theorists in the field of chemical reaction dynamics.

\section{EXPERIMENTAL CONSIDERATIONS}

The experiments were carried out using the highresolution monochromatized VUV undulator synchrotron source of the Chemical Dynamics Beamline ${ }^{18}$ at the ALS. This VUV synchrotron source consists of a 10-cm-period undulator, a gas harmonic filter, and a high-resolution $6.65 \mathrm{~m}$ off-plane Eagle mounted scanning monochromator. All details concerning these elements have been described previously. The ALS ring was operated in the multibunch mode with a dark gap of 104 ns. ${ }^{15}$ Neon was used in the harmonic gas filter to suppress the higher undulator harmonics with photon energies greater than $21.565 \mathrm{eV}$. A 2400 lines $/ \mathrm{mm}$ grating (dispersion $=0.64 \AA / \mathrm{mm}$ ) was used to disperse the first harmonic of the undulator VUV beam with entrance/exit slits sizes in the range of 30-100 $\mu \mathrm{m}$. The dispersed VUV radiation emerging from the monochromator was focused into the photoionization center of the octopole-quadrupole photoionization apparatus.

\section{A. The octopole-quadrupole photoionization apparatus}

Figure 1 shows the schematic diagram of the octopolequadrupole photoionization apparatus implemented at the Chemical Dynamics Beamline of the ALS. This apparatus combines guided-ion beam mass spectrometry ${ }^{24}$ and PFIPESICO methods for state- or energy-selected ion-molecule reaction studies. This apparatus consists of, in sequential order, a PFI-PEPICO ion source for the internal state selection of reactant ions, ${ }^{17}$ an interleaved-comb wire ion gate lens ${ }^{19,20}$ (10) for rejection of false coincidence ions, a dual-rf octopole

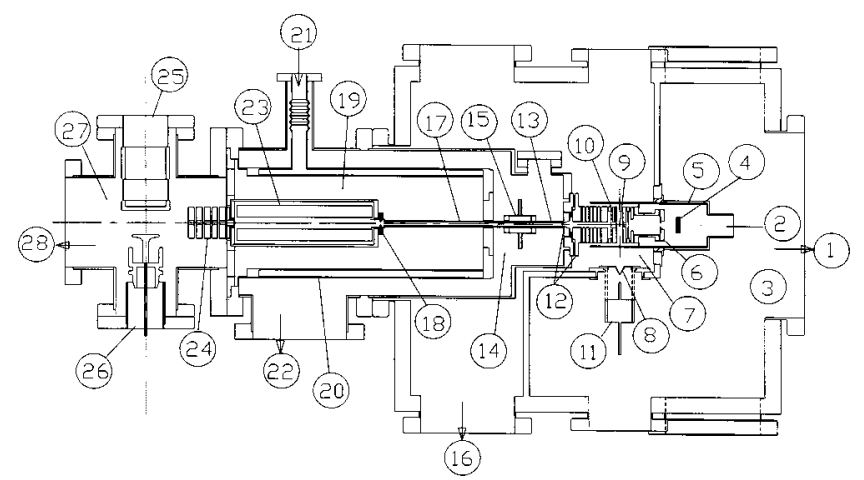

FIG. 1. Schematic diagram for the octopole-quadrupole photoionization apparatus. (1) To $2000 \mathrm{~L} / \mathrm{s}$ turbomolecular pump, (2) to $300 \mathrm{~L} / \mathrm{s}$ turbomolecular pump, (3) molecular beam source chamber, (4) dual MCP photoelectron detector, (5) $\mu$-metal shield, (6) electron TOF spectrometer, (7) photoionization chamber, (8) skimmer, (9) photoionization center, (10) wired ion gate lens or ion gate, (11) nozzle and nozzle holder, (12) ion injection lenses, (13) short $\mathrm{rf}$ octopole $(8.6 \mathrm{~cm}),(14)$ reaction chamber, (15) reaction gas cell, (16) to $800 \mathrm{~L} / \mathrm{s}$ turbomolecular pump, (17) long rf octopole $(19.6 \mathrm{~cm}),(18)$ entrance ion lenses for QMS, (19) QMS chamber, (20) liquid nitrogen cooled wall, (21) liquid nitrogen inlet, (22) to $800 \mathrm{~L} / \mathrm{s}$ turbomolecular pump, (23) QMS, (24) exit ion lenses for QMS, (25) + (26) Daly ion detector, (27) detector chamber, and (28) to $600 \mathrm{~L} / \mathrm{s}$ turbomolecular pump.

ion guide reaction gas cell $[(13)+(15)+(17)]$ for efficient collection of product ions, a quadrupole mass spectrometer (QMS) (23) for reactant and product mass identifications, and a Daly scintillation detector ${ }^{25}[(25)+(26)]$ for ion detection. The QMS chamber wall (20) can be cooled by liquid nitrogen, which increases the pumping speed for condensable gases. The rf-octopole-quadrupole arrangement is similar to that described previously. ${ }^{2-4,6}$ Because the purity of reactant ions formed by photoionization is high, reactant ions can be sent into the rf octopole ion guide reaction gas cell [(15), nominal length $=5 \mathrm{~cm}]$ without mass filtering. The dual rf-octopole ion guide consists of a short [(13), length $=8.64 \mathrm{~cm}]$ and a long [(17), length $=19.55 \mathrm{~cm}]$ octopole units. Both octopoles, constructed of eight electropolished 316 stainless steel rods (diameter $=0.2 \mathrm{~cm}$ ), are symmetrically spaced on an inscribed circle with a diameter of $0.6 \mathrm{~cm}$. These octopole units are powered by a single rf-power supply, but with separate dc potentials for the two octopole units. The reaction gas cell (15) encompasses the last part of the short rf octopole. Because different dc potentials can be applied to the long and short octopoles, slow primary product ions, such as charge transfer product ions, can be extracted from the reaction cell to minimize secondary reactions between slow product ions and neutral reactant molecules in the gas cell. This arrangement also enables TOF analysis of the axial and radial velocity distributions of product ions. ${ }^{24}$

To facilitate the description below, we show in Fig. 2 a magnified view around the photoionization region, showing the detailed design of the PFI-PE detector, the ion lens system, and the dual rf-octopole ion guide reaction gas cell, the effusive beam inlet, and the supersonic beam production system. The PFI-PE detector, which consists of a set of electron lenses (6), a dual microchannel plate (MCP) (4), and a $\mu$-metal shield (5), has the same design as that used in previous PFI-PEPICO measurements. ${ }^{17}$ The photoionization 


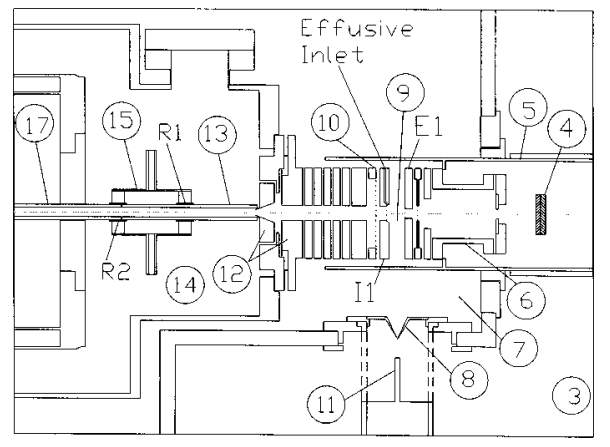

FIG. 2. Magnified view of the PFI-PE TOF spectrometer $(4)+(5)+(6)$, photoionization region (9) defined by repeller plate I1 and E1, wired ion gate lens or ion gate (10), ion injection lenses (12), short rf octopole (13), reaction gas cell (15), long rf octopole (17), and the molecular beam production system, which includes the skimmer (8) and the nozzle and nozzle holder (11) assembly. Note that the neutral gas sample for photoionization can also be introduced as an effusive beam through a channel drilled through the repeller plate I1. R1 and R2 situated at the entrance and exit of the reaction gas cell are ring electrodes.

center (9) is located at the center of two repeller plates, I1 and E1, which are spaced $1.0 \mathrm{~cm}$ apart. The wired ion gate lens (10) is situated next to the ion repeller plate I1. The fabrication and detailed performance of the wired ion gate lens for the rejection of false coincidence ions is described below. The six aperture ion lenses following the wired ion gate lens are used for transporting the reactant ions formed in the PI/PEX region toward the short rf octopole (13). The two ion injection lenses (12) before the entrance of the rf octopole (13) focus the reactant ions into the short rf octopole. The ring electrodes ${ }^{24} \mathrm{R} 1$ and $\mathrm{R} 2$ situated at the entrance and exit of the reaction gas cell are also shown in Fig. 2. A positive dc potential with respect to the octopole applied to $\mathrm{R} 1$ can be used to generate a small positive potential barrier within the octopole. This "penetration potential barrier" can reflect backscattered charge transfer product ions toward the QMS. The second electrode R2 can be used to offset a possible electrostatic barrier at the junction of the two octopoles by applying a negative potential.

Reactant ions are prepared by photoionization of a neutral precursor gas introduced into the photoionization region as an effusive beam. The effusive gas beam is formed by flowing the neutral precursor gas through a channel (diameter $=0.020$ in.) drilled through the repeller plate I1. The exit of the channel points at and is located $\approx 0.5 \mathrm{~cm}$ from the photoionization center (9). The neutral precursor gas can be introduced in the form of a supersonic beam by expansion through the stainless steel nozzle (11). This beam is skimmed by a conical skimmer (8) prior to intersecting the monochromatic VUV beam at $90^{\circ}$ in the photoionization region.

The octopole-quadrupole photoionization apparatus is partitioned into four differentially pumped chambers, namely, the molecular beam source chamber (3), the photoionization chamber (7), the reaction chamber (14), and the quadrupole chamber (19), which are evacuated by turbomolecular pumps with pumping speeds of 2600, 1400, 1000, and $600 \mathrm{~L} / \mathrm{s}$, respectively. Note that the detector chamber (27) and the quadrupole chamber (19) are connected and are evacuated by the same $(600 \mathrm{~L} / \mathrm{s})$ turbomolecular pump. For the electron microchannel plate (MCP) to operate properly, it is necessary to maintain a sufficiently good vacuum $\left(<10^{-6}\right.$ Torr $)$ in the vicinity of the electron MCP. For this reason, the end of the PFI-PE spectrometer is connected to the inlet of a $300 \mathrm{~L} / \mathrm{s}$ turbomolecular pump by a flexible bellow. The neutral reactant gas pressure (1.5-2.5 $\times 10^{-4}$ Torr) used in the reaction gas cell (15) is monitored by an MKS Baratron. During the experiment, the pressures in the photoionization chamber and reaction chamber were maintained at $\leqslant 2 \times 10^{-6}$ Torr and the QMS and detector chamber was $\leqslant 10^{-7}$ Torr. When the neutral precursor gas was introduced into the photoionization region by using the supersonic beam source, the beam source chamber was maintained at $\approx 10^{-4}$ Torr.

The data acquisition for absolute cross-section measurements is fully automated and controlled by a combination of standard data acquisition electronics, computer interfaces, LabView modules and a software suite ${ }^{26}$ developed previously.

\section{B. Absolute total cross-section measurements}

The cross-sections measurement for $\mathrm{Ar}^{+}\left({ }^{2} \mathrm{P}_{3 / 2,1 / 2}\right)+\mathrm{D}_{2}$ and $\mathrm{O}_{2}{ }^{+}\left(v^{+}=3\right)+\mathrm{Ar}$ described below are test experiments to illustrate the basic performance of the octopolequadrupole photoionization apparatus. In these experiments, an appropriate dc voltage difference $(\Delta V)$ was applied between the repeller plates E1 and I1 to extract reactant ions (formed by photoionization in the PI/PEX region) toward the reaction gas cell, where the ion-molecule reactions take place:

Accurate absolute total cross sections for reaction (3) have been reported ${ }^{27}$ previously over a wide collision energy range with a mixture of $\mathrm{Ar}^{+}$spin-orbit states prepared by electron impact:

$$
\operatorname{Ar}^{+}\left({ }^{2} \mathrm{P}_{3 / 2,1 / 2}\right)+\mathrm{D}_{2} \rightarrow \operatorname{ArD}^{+}+\mathrm{D} .
$$

These cross-sections for the formation of $\mathrm{ArD}^{+}(\bullet)$ in the laboratory kinetic energy $\left(E_{\text {lab }}\right)$ range of $0.2-10 \mathrm{eV}$ are compared in Fig. 3 to those $(+)$ measured using the present octopole-quadrupole ion-molecule reaction apparatus of Fig. 1. In both the electron impact and in our PI experiments, the reactant $\mathrm{Ar}^{+}$ions are expected to be formed in a $\operatorname{Ar}^{+}\left({ }^{2} \mathrm{P}_{3 / 2}\right): \operatorname{Ar}^{+}\left({ }^{2} \mathrm{P}_{1 / 2}\right)$ ratio of $2: 1$. The generally good agreement between the two experiments indicates that the effective length of the reaction gas cell can be taken to be its actual length of $5.0 \mathrm{~cm}$. The reactant $\operatorname{Ar}^{+}\left({ }^{2} \mathrm{P}_{3 / 2,1 / 2}\right)$ ions are prepared by electron-impact ionization in the previous experiment, whereas the reactant $\operatorname{Ar}^{+}\left({ }^{2} \mathrm{P}_{3 / 2,1 / 2}\right)$ ions in the present experiment are formed by photoionization at a photon energy above the IE for the formation of $\operatorname{Ar}^{+}\left({ }^{2} \mathrm{P}_{1 / 2}\right)$. In both experiments, the reactant $\mathrm{Ar}^{+}$ions were expected to be formed in $\operatorname{Ar}^{+}\left({ }^{2} \mathrm{P}_{3 / 2}\right): \mathrm{Ar}^{+}\left({ }^{2} \mathrm{P}_{1 / 2}\right)$ ratio of 2:1. This test experiment also shows that the kinetic energy resolution achieved using the present octopole-quadrupole photoionization apparatus is capable of measuring reaction cross sections down to thermal collision energies. Because the photon spot size at the photoionization region is $\Delta l=0.3 \mathrm{~mm}$, the 


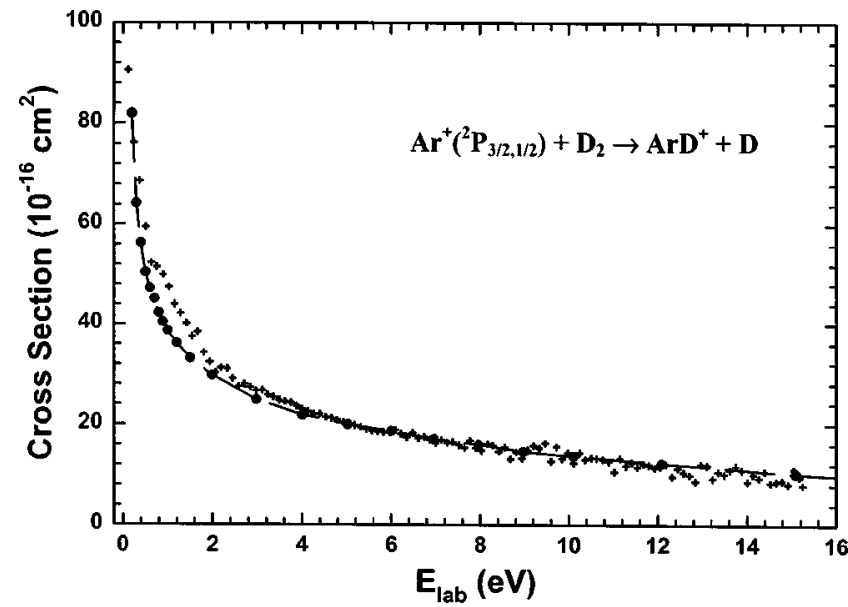

FIG. 3. Absolute total cross sections for reaction $\operatorname{Ar}^{+}\left({ }^{2} \mathrm{P}_{3 / 2,1 / 2}\right)+\mathrm{D}_{2}$ $\rightarrow \mathrm{ArD}^{+}+\mathrm{D}$ in the $E_{\text {lab }}$ range of $0.03-10 \mathrm{eV}$. (-•): reported in Ref. 27 and $(+)$ : obtained using the octopole-quadrupole photoionization apparatus shown in Fig. 1.

resolution for $E_{\text {lab }}\left(\Delta E_{\text {lab }}\right)$ is predicted to be $\Delta E_{\text {lab }}=(\Delta l / l)$ $\times(\Delta V)=(0.03) \Delta V$. Using the $\Delta V$ value of $1 \mathrm{~V}$, a $\Delta E_{\text {lab }}$ value of $\approx 0.03 \mathrm{eV}$ can be readily attained.

We have also examined the collision-induced dissociation and charge transfer cross sections for the reaction of $\mathrm{O}_{2}{ }^{+}+\mathrm{Ar}$ (not shown here) with reactant $\mathrm{O}_{2}{ }^{+}$prepared in the $v^{+} \leqslant 3$ state by photoionization. These cross-section curves are similar to those reported in Ref. 28. Despite the low cross sections at the thresholds, the appearance energies observed for $\mathrm{O}^{+}$and $\mathrm{Ar}^{+}$were found to be consistent with their thermochemical thresholds, demonstrating that reaction cross sections down to the range of $10^{-18}-10^{-19} \mathrm{~cm}^{2}$ can be measured using the octopole-quadrupole photoionization apparatus.

\section{Fabrication and performance of the fast ion gate}

As pointed out above, the success of the PFI-PESICO TOF measurements for state-selected ion-molecule reactions depends critically on the novel application of the fast ion gate $^{19,20}(10)$. The ion gate consists of an array of parallel wires separated by $0.0125 \mathrm{in}$. The wires are electrically connected in an interleaved manner such that neighboring wires are electrically insulated from each other. The parallel wire assembly was fabricated by winding of a 0.0008 in. gold plated tungsten wire on a 4 in. $\times 5$ in. rectangular circuit board with a 3 in. $\times 4$ in. open central area, as shown in Fig. 4 , where the outer gray regions are the common area (where a floating voltage can be applied), the upper and lower T-shaped regions are conducting areas for connection to the even and odd sets of tungsten wires, respectively. The upper and lower light-gray strips between the T-shaped region and the open central region are areas for applying epoxy to fix the positions of the odd and even sets of wires, respectively.

The fabrication was made on an assembly of eight identical circuit boards (Fig. 4), which were mounted to the faces of an aluminum octagon. The axle of the octagon was placed in the chuck of a lathe. The wire spacing of 0.0125 in. was determined by the 80 threads/in. pitch on the lathe. A pully

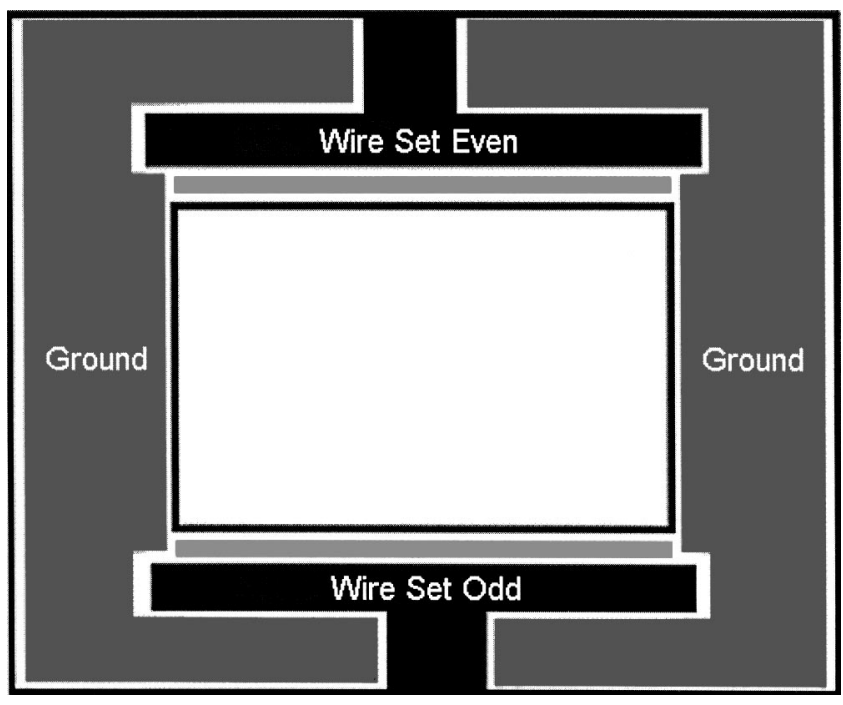

FIG. 4. The 4 in. $\times 5$ in. rectangular circuit board with a 3 in. $\times 4$ in. open area. The outer gray region is the grounding area, the black upper and lower T-shaped regions are conducting areas for connection to the even and odd tungsten wires, respectively. The light-gray strips just above and below the open area are areas for applying epoxy to fix the positions of the odd and even sets of wires

system was used to hang a fixed mass on the wire feed to provide a constant tension while being wound. A piece of double sided tape was applied to each vertex of the octagon in order to prevent the wires from slipping on the circuit board. Once the wire was completely wound, two beads of epoxy were applied (to the strip regions located at the upper and lower edges of the open central area as shown in Fig. 4) to fix the wires on each of the frames. The frames could then be removed from the wheel. The next step involves cutting every other wire between the epoxy and the conductive strip. To simplify this procedure, the wired circuit board was placed on a micrometer-adjust translation stage, and a splined jewelers drill was used, with a small bit sharpened to a chisel point (the drill was not rotating). The construction of the wired ion gate lens is completed by soldering the remaining wires on each side to the conductive strip.

By applying a positive and negative potential of across the two sets of wires, a strong and highly local electric field can be generated between adjacent wires. Ions can be effectively deflected away from the beam path as they pass through the grid. Due to the small distance between adjacent wires, a small potential difference is sufficient for deflecting the false coincidence ions. Because low kinetic energy ions are more easily deflected than fast ions, and because it is desirable to reject the false ions when they are spatially compact, we have placed the ion gate early in the ion trajectory, namely next to the ion repeller plate I1. Due to the short range nature of the wire deflection fields, the ion gate can be used as a fast "ion gate" to pass the coincidence PFI-PI at a fixed delay with respect to the detection of a PFI-PE by switching off the wire deflection field.

In the present experiment, there are two sources of false coincidence ions. One contribution arises from direct VUV photoionization. The time distribution of this coincidence background as seen by the ion detector is determined by the 
pattern of VUV micropulses emitted by the ALS synchrotron ring. One ALS synchrotron period of $656 \mathrm{~ns}$ consists of a light-on interval of $552 \mathrm{~ns}$ [276 micropulses (width $=50 \mathrm{ps)}$ with uniform separations of $2 \mathrm{~ns}$ ] and a dark gap of 104 ns. ${ }^{17,18}$ The coincidence TOF measurements show that the ion TOF widths observed are in the range of $1-5 \mu \mathrm{s}$. Thus, the ion arrivals at the ion detector are dispersed into the temporal regions associated with the dark gaps, making the time distribution of false coincidence ions more uniform. The PFI-PIs are formed by PFI in the dark gap using a 200 ns electric field pulse. False coincidence events can also occur if either the PFI-PE or PFI-PI is lost on the way to the electron or ion detector. The distribution of this second source of false coincidence ions should center in the temporal regions associated with the dark gaps. Both the contributions by direct ionization and by PFI events are expected to give rise to a wave-like pattern of the false coincidence background with the period equal to that of the ALS ring (656 ns). This expectation was confirmed by previous PFIPEPICO measurements. ${ }^{17}$ However, the large ion dispersion of 1-5 $\mu$ s as observed by coincidence ion TOF measurements in this experiment has greatly smoothened the wavelike pattern, yielding a near time-independent false coincidence counting rate when average over a temporal range of a few $\mu$ s. The near uniform time distribution of false coincidence ions is confirmed by PFI-PEPICO ion TOF spectra presented below. Since the PFI pulse is applied at a repetition rate of $1.53 \mathrm{MHz}$ and the PFI-PE intensity is generally $<3000$ counts/s, the probability of forming a PFI-PI by one PFI pulse is $<2 \times 10^{-3}$. Considering the fact that the total ionization rate is $\leqslant 10^{5}$ ions/s, the average false coincidence ion produced in the time window of $200 \mathrm{~ns}$ is $<2$ $\times 10^{-2}$ ions. This analysis shows that the probability of producing a PFI-PI and a false coincidence ion together in the same time window of $200 \mathrm{~ns}$ is negligibly small. The statistical or random nature of the formation of PFI-PIs and false coincidence ions has made possible the PFI-PEPICO and PFI-PESICO measurements. ${ }^{17}$

In the experiments described below, the ion gate is floated at a dc voltage (applied to all wires) in the range of -2.5 to $-8 \mathrm{~V}$. This de potential can be determined by maximizing the ion transmission. The deflecting field resulting from the positive and negative potential does not affect the trajectories of ions at a distance of a few times of the wire spacing from the ion gate because the alternate positive and negative potentials seen by these ions are exactly canceled. The detection of a PFI-PE by the electron detector signifies the production of an energy-selected ion, i.e., PFI-PI, at the photoionization region. In order to select this one ion and reject all the nonenergy selected ions, the strategy is to open the fast ion gate for the minimum time necessary to let this ion pass, while rejecting all other ions. As described below, typical open times for the ion gate are 200-300 ns. For a PFI-PE trigger rate of $\approx 1000$ counts/s, the opening time per second for the ion gate is $\approx 2-3 \times 10^{-7} \mathrm{~s}$. Assuming that background false coincidence ions are generated uniformly in time, the false coincidence signal should be reduced by a factor of $\approx 2-3 \times 10^{-7}$ by using the wired ion gate scheme.

\section{PFI-PEPICO measurements}

The experimental conditions and dark-gap PFI scheme used for PFI-PE detection and PFI-PI extraction are similar to those described in previous PFI-PEPICO measurements. ${ }^{17}$ Briefly, a nominal dc field of zero $\mathrm{V} / \mathrm{cm}$ was set across the photoionization region. The application of a PFI and ion extraction field pulse $(8 \mathrm{~V} / \mathrm{cm}$, width $=200 \mathrm{~ns})$ was delayed by $\approx 10 \mathrm{~ns}$ with respect to the beginning of the 104 ns dark gap. The field was achieved by applying an electric pulse of -4.0 $\mathrm{V}$ at $\mathrm{I} 1$ and $+4.0 \mathrm{~V}$ at $\mathrm{E} 1$. As pointed out in previous studies, the employment of a PFI pulse with the duration of $200 \mathrm{~ns}$ is necessary for efficient extraction of the PFI-PI.

The PFI-PEPICO TOF peak for $\mathrm{Ar}^{+}$observed using the $10 \mathrm{~V} / \mathrm{cm}(-5.0 \mathrm{~V}$ at $\mathrm{I} 1$ and $+5.0 \mathrm{~V}$ at E1), $200 \mathrm{~ns}$ long pulsed extraction field and a linear TOF mass spectrometer with an Ar supersonic beam sample has a FWHM of $\approx 300$ ns. ${ }^{17}$ Figure 5(a) shows the experimental PFI-PEPICO TOF spectrum for $\operatorname{Ar}^{+}\left({ }^{2} \mathrm{P}_{1 / 2}\right)$ formed by photoionization of an effusive $\mathrm{Ar}$ beam at $298 \mathrm{~K}$ without using the ion gate. The observed FWHM for the $\operatorname{Ar}^{+}\left({ }^{2} \mathrm{P}_{1 / 2}\right)$ TOF peak of Fig. 5(a) is $\approx 4 \mu \mathrm{s}$ (FWHM). Thus, the false coincidence background for the PFI-PEPICO measurement of $\operatorname{Ar}^{+}\left({ }^{2} \mathrm{P}_{1 / 2}\right)$ (measured under the coincidence TOF peak) is expected to be $\approx 13$ times higher than that observed in the previous molecular beam TOF measurement. The intensity of false coincidences in the spectrum of Fig. 5(a) is a factor of 4 higher than the coincidence peak. Figures 5(b) and 5(c) show the PFI-PEPICO TOF spectra for $\operatorname{Ar}^{+}\left({ }^{2} \mathrm{P}_{3 / 2}\right)$ and $\operatorname{Ar}^{+}\left({ }^{2} \mathrm{P}_{1 / 2}\right)$, respectively, obtained using the ion gate. Here, the ion gate (duration $=200 \mathrm{~ns}$ ) was opened twice for each PFI-PE trigger pulse. The first ion gate was opened at the correct delay of $4.7 \mu \mathrm{s}$ with respect to the PFI-PE trigger pulse to pass the true PFIPIs, whereas the second ion gate was opened after an arbitrary delay of $25 \mu \mathrm{s}$ with respect to the first ion gate. Thus, the first TOF peak measures mostly the intensity of true coincidence ions, whereas the second TOF peak [marked by arrow in Figs. 5(a) and 5(b)] gives only the false coincidence ions. The true coincidence counts can be obtained by taking the difference in counts of the first and second TOF peaks. For the spectra shown in Figs. 5(b) and 5(c), the center bias voltage and the (positive, negative) voltages (with respect to the center bias voltage) applied to the (even, odd) sets of wires of the ion gate are $-2.5 \mathrm{~V}$ and $(+2.5 \mathrm{~V},-2.5 \mathrm{~V})$, respectively. Note that the background level observed in Figs. 5(b) and 5(c) is not zero. As expected, the background level for the $\operatorname{Ar}^{+}\left({ }^{2} \mathrm{P}_{1 / 2}\right)$ band is higher than that for the $\operatorname{Ar}^{+}\left({ }^{2} \mathrm{P}_{3 / 2}\right)$ band. This constant background also depends on the (positive, negative) voltages (or the deflection field) applied to the wired ion gate for shutting off the false background ions.

Figures 6(a), 6(b), and 6(c) compare the PFI-PEPICO TOF spectra obtained using a constant bias voltage of -2.5 $\mathrm{V}$, but setting the relative voltages on the wires to \pm 2.5 , \pm 3.0 , and $\pm 3.5 \mathrm{~V}$ applied to the odd and even sets of wires, respectively. As shown in these figures, the constant background of the PFI-PEPICO spectrum decreases as the magnitude of the (positive, negative) voltage increases, i.e., the deflection field between adjacent wires is increased, resulting 


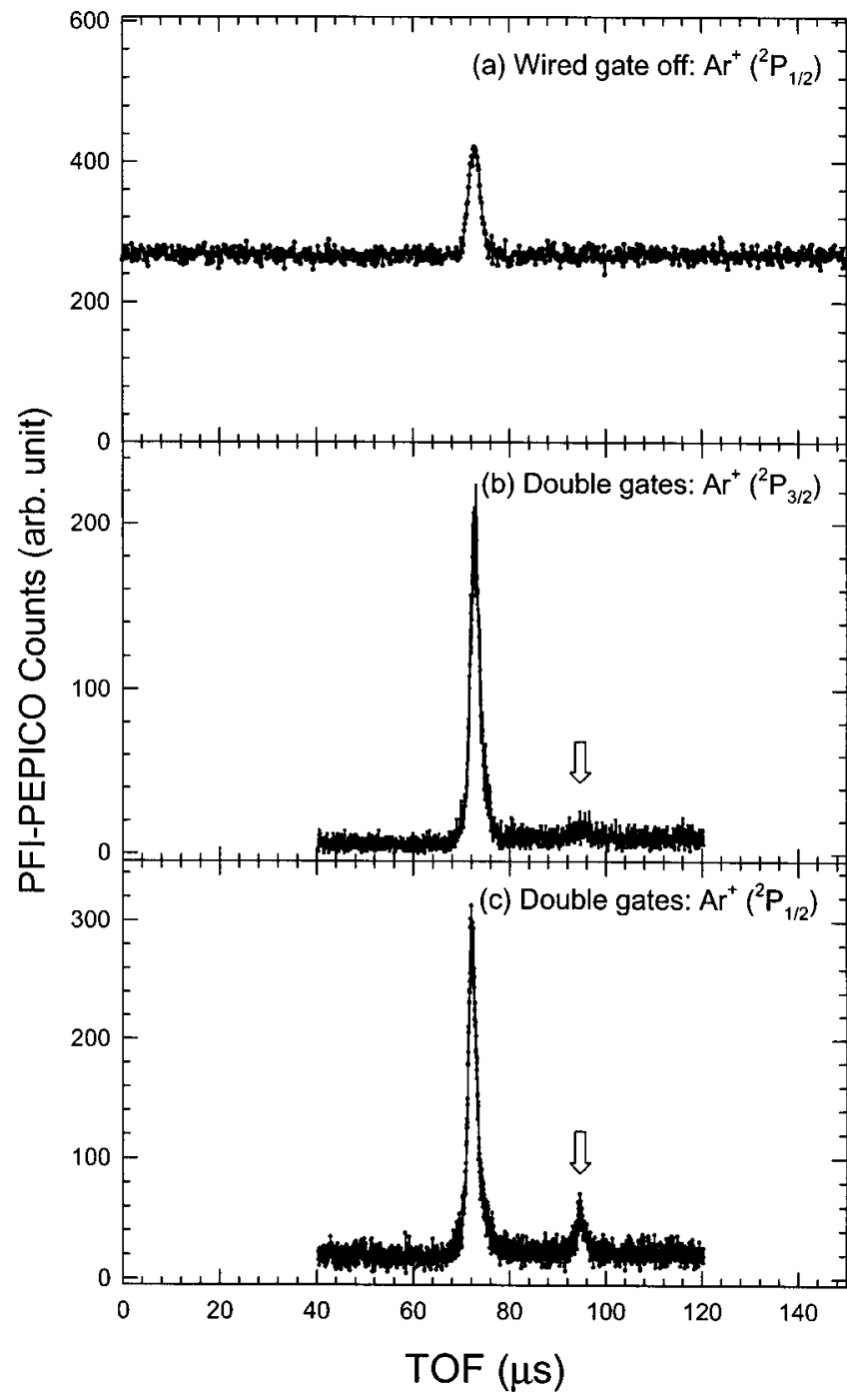

FIG. 5. (a) PFI-PEPICO bands for $\operatorname{Ar}^{+}\left({ }^{2} \mathrm{P}_{3 / 2}\right)$ obtained without using the wired gate. (b) PFI-PEPICO band for $\operatorname{Ar}^{+}\left({ }^{2} \mathrm{P}_{1 / 2}\right)$ obtained using the ion gate. (c) PFI-PEPICO band for $\mathrm{Ar}^{+}\left({ }^{2} \mathrm{P}_{1 / 2}\right)$ obtained using the ion gate. The ion gate (duration $=200 \mathrm{~ns}$ ) was opened twice for each PFI-PE trigger pulse. The first ion gate was opened at the correct delay $(4.70 \mu \mathrm{s})$ to pass the true PFI-PIs and the second ion gate was opened after an arbitrary delay of $25 \mu \mathrm{s}$ with respect to the first ion gate. The first TOF peaks measure mostly the intensities for true coincidence ions, whereas the second TOF peaks (marked by arrows) give only the false coincidence ions. That is, the true coincidence counts equal to the difference in counts of the first and second TOF peaks. The dc bias and deflection voltages of the ion gate are $-2.5 \mathrm{~V}$ and $\pm 2.5 \mathrm{~V}$, respectively.

in more effective deflection of the false coincidence ions. As expected, the appropriate deflection voltage for shutting off the false coincidence ions also depends on the ion energy at (or the center bias dc voltage applied to) the ion gate. Once the conditions for selecting state-selected reactant ions are determined by maximizing the intensity of coincidence PFIPIs from the photoionization region to the ion detector, the voltage settings of all the ion lenses situated between the photoionization region and the ion injection lenses can be fixed throughout the PFI-PESICO measurement to be described below. Because the photoionization center is always maintained (nominally) at ground potential, $E_{\text {lab }}$ of the ionmolecule reaction under investigation is thus controlled by the dc bias potential of the short rf octopole, where the reac-

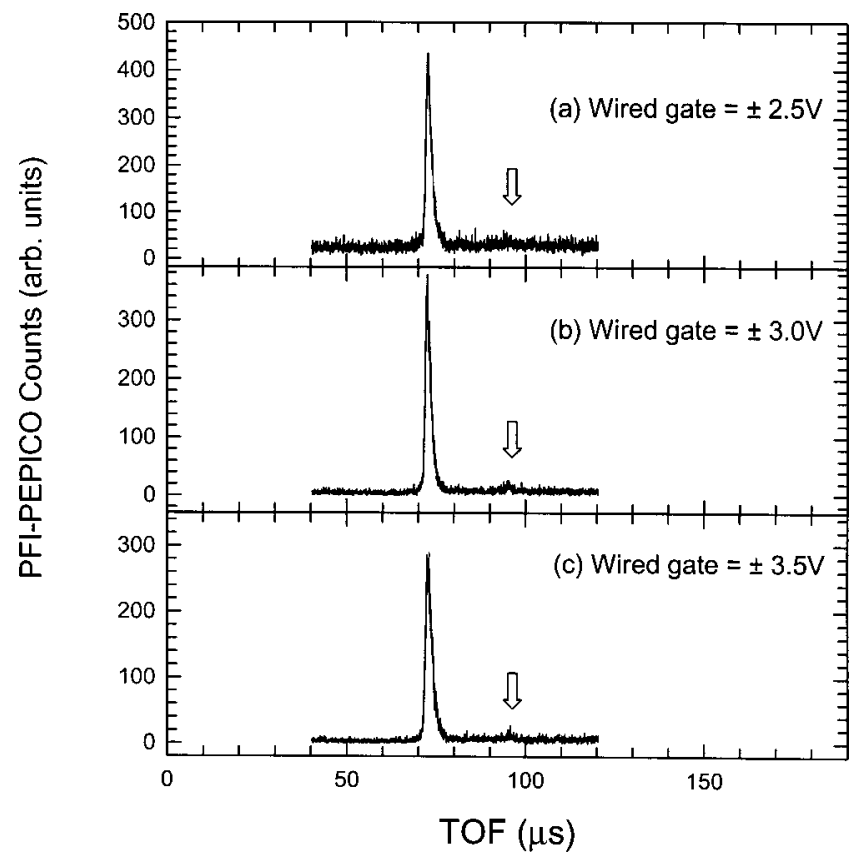

FIG. 6. PFI-PEPICO spectra for $\operatorname{Ar}^{+}\left({ }^{2} \mathrm{P}_{3 / 2}\right)$ obtained using the ion gate. Delay of the ion gate $=4.70 \mu \mathrm{s}$. The second ion gate was opened after an arbitrary delay of $25 \mu$ s with respect to the first ion gate. Floating dc or center bias voltage $=-2.5 \mathrm{~V}$. Ion gate duration $=200 \mathrm{~ns}$. The (positive, negative) voltages are: (a) $(+2.5 \mathrm{~V},-2.5 \mathrm{~V}) ;(\mathrm{b})(+3.0 \mathrm{~V},-3.0 \mathrm{~V})$; and (c) $(+3.5 \mathrm{~V},-3.5 \mathrm{~V})$. The second TOF peaks that measure the false coincidence ion counts are marked by arrows.

tion gas cell is situated. The efficiency of ion injection into the reaction gas cell (or short rf octopole) is mostly governed by the injection lenses (12) shown in Figs. 1 and 2.

Figure 7 compares the PFI-PE band for $\mathrm{Ar}^{+}\left({ }^{2} \mathrm{P}_{1 / 2}\right)$ (open circles) with the PFI-PEPICO band for $\operatorname{Ar}^{+}\left({ }^{2} \mathrm{P}_{1 / 2}\right)$ (solid squares) obtained using the differential-pulse-ion-gate PFIPEPICO scheme. The good agreement between the PFI-PE and PFI-PEPICO bands can be taken as confirmation of the differential-pulse-ion-gate PFI-PEPICO scheme. However, the counting rate for the PFI-PEPICO measurement is nearly

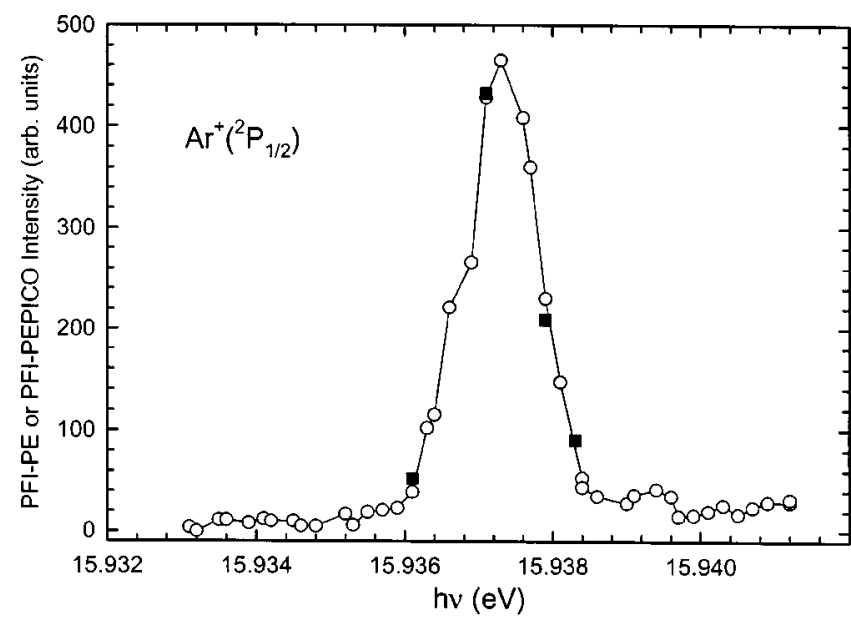

FIG. 7. Comparison of the PFI-PE band for $\operatorname{Ar}^{+}\left({ }^{2} \mathrm{P}_{1 / 2}\right)$ (open circles) with the PFI-PEPICO band for $\mathrm{Ar}^{+}\left({ }^{2} \mathrm{P}_{1 / 2}\right)$ (solid squares) measured using the differential-pulse-ion-gate scheme. Note that the PFI-PEPICO band was measured only at four distinct energies and was scaled to match the PFI-PE band. 

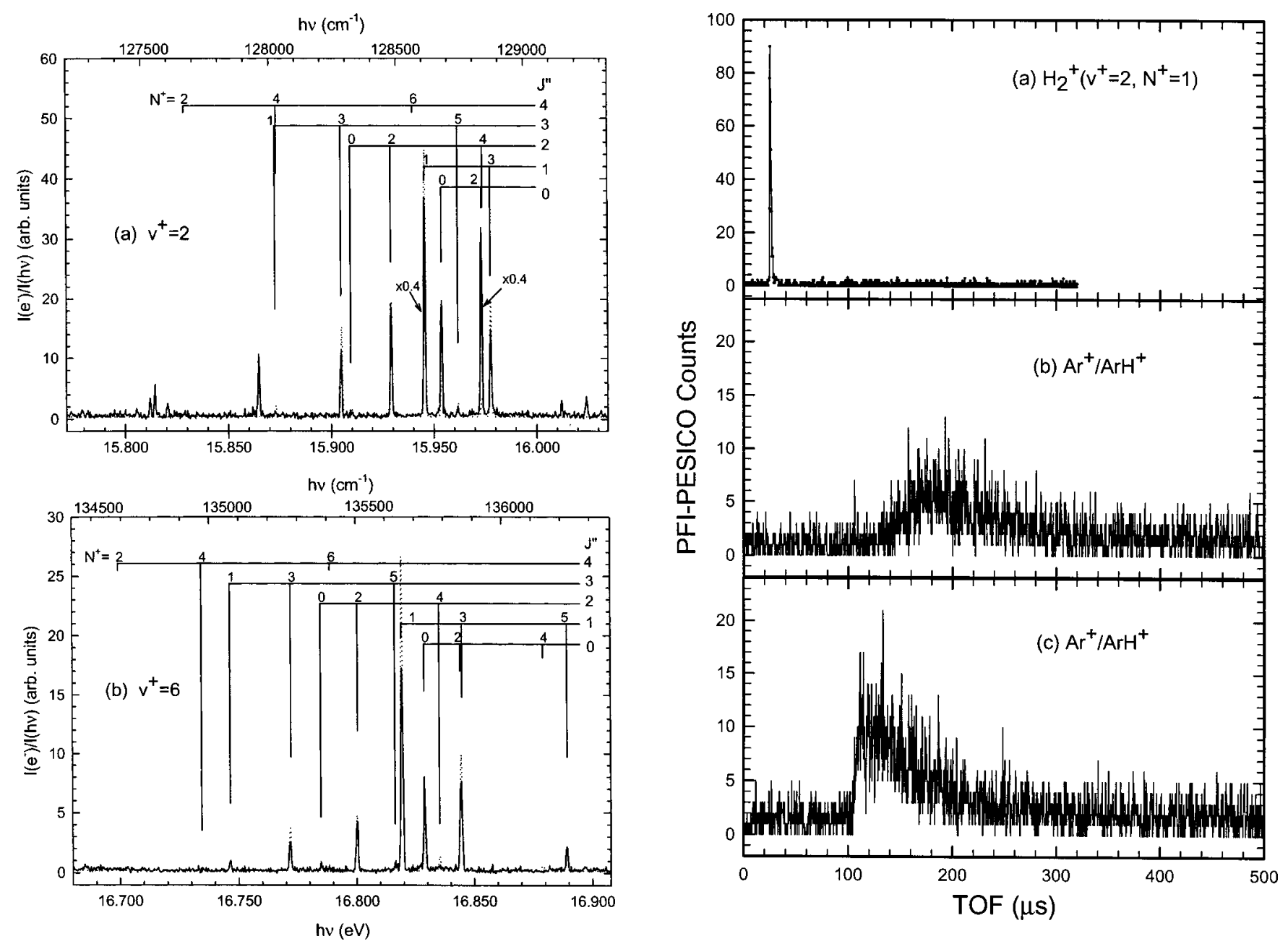

FIG. 8. (a) Rotationally resolved PFI-PE vibrational band for $\mathrm{H}_{2}{ }^{+}\left(v^{+}\right.$ $=2)$. Note that the intensities for $\left(N^{+}, J^{\prime \prime}\right)=(1,1)$ and $(2,0)$ transitions are reduced by a factor of 0.4 . (b) Rotationally resolved PFI-PE vibrational band for $\mathrm{H}_{2}^{+}\left(v^{+}=6\right)$.

40-fold lower than that of the PFI-PE measurement, indicating the low transmission for $\mathrm{Ar}^{+}$.

\section{E. PFI-PESICO measurements}

We have obtained absolute integral cross sections for the state-selected ion-molecule reaction $\mathrm{H}_{2}{ }^{+}\left(v^{+}=0-17, N^{+}\right.$ $=1)+\mathrm{Ar}$ at specific $E_{\text {c.m. }}$ values using the PFI-PESICO method. ${ }^{23}$ Selected results of this reaction are described here to illustrate the performance of the differential-pulse-ion-gate PFI-PESICO scheme. Previous experiments have only provided absolute integral cross sections for the vibrational state-selected reaction of $\mathrm{H}_{2}{ }^{+}\left(v^{+} \leqslant 4\right)+$ Ar. ${ }^{3,5}$ To our knowledge, the cross-section measurements for the rotational and vibrational state-selected reaction of $\mathrm{H}_{2}{ }^{+}\left(v^{+}, N^{+}\right)+\mathrm{Ar}$ have not been made.

The rotationally resolved vibrational PFI-PE bands for $\mathrm{H}_{2}{ }^{+}\left(v^{+}=0-18\right)$ have been obtained previously. ${ }^{15,16,29,30} \mathrm{As}$ an example, we show in Figs. 8(a) and 8(b) the PFI-PE bands for $\mathrm{H}_{2}{ }^{+}\left(v^{+}=2, N^{+}\right)$and $\mathrm{H}_{2}^{+}\left(v^{+}=6, N^{+}\right)$, respectively, which reveal well-resolved rotational transitions as marked in the figures. We note that Figs. 8(a) and 8(b) have the same intensity scale. Due to the rotational distributions of ortho-

FIG. 9. (a) PFI-PEPICO TOF spectrum for reactant $\mathrm{H}_{2}{ }^{+}\left(v^{+}=2, N^{+}=1\right)$ (accumulation time $=2 \mathrm{~min}$ ). (b) PFI-PESICO TOF spectrum for product $\mathrm{Ar}^{+} / \mathrm{ArH}^{+}$at $E_{\text {lab }}=2.5 \mathrm{eV}$ obtained by setting the de potential of the long octopole $0.4 \mathrm{~V}$ lower than that of the short octopole (accumulation time $=50 \mathrm{~min}$ ). (c) PFI-PESICO spectrum for $\mathrm{Ar}^{+} / \mathrm{ArH}^{+}$at $E_{\mathrm{lab}}=2.5 \mathrm{eV}$ obtained by setting the dc potential of the long octopole $-2 \mathrm{~V}$ lower than that of the short octopole (accumulation time $=60 \mathrm{~min}$ )

and para-hydrogen at $298 \mathrm{~K}$ and selection rules for photoionization, the $N^{+}=0,1,2$, and 3 rotational states for many $\mathrm{H}_{2}^{+}\left(v^{+}\right)$states, as shown in Figs. 8(a) and 8(b) for $v^{+}=2$ and 6 , can be prepared with good intensities by photoionization of normal $\mathrm{H}_{2}$ at $298 \mathrm{~K}$.

Figure 9(a) shows the PFI-PEPICO TOF spectrum for reactant $\mathrm{H}_{2}{ }^{+}\left(v^{+}=2, N^{+}=1\right)$ recorded at $E_{\mathrm{lab}}=2.5 \mathrm{eV}$ using the differential-pulse-ion-gate scheme described above. The first ion gate (duration $=300 \mathrm{~ns}$ ) is delayed by $1.50 \mu$ s with respect to the PFI-PE pulse and the second ion gate (duration $=300 \mathrm{~ns}$ ) is set at $150 \mu$ s with respect to the first ion gate. The TOF peak observed in Fig. 9(a) represents the true coincidence $\mathrm{H}_{2}{ }^{+}$ions [or $\mathrm{H}_{2}{ }^{+}\left(v^{+}=2, N^{+}=1\right)$ PFI-PIs] and false coincidence $\mathrm{H}_{2}{ }^{+}$ions transmitted through the ion gate. The second TOF peak, which samples only the false coincidences, is not discernible. This observation indicates that the false coincidence ion intensity under the (first) TOF peak of Fig. 9(a) is negligible. The PFI-PESICO TOF spectrum shown in Fig. 9(b) is for the sum of product $\mathrm{Ar}^{+}$and $\mathrm{ArH}^{+}\left(\mathrm{Ar}^{+} / \mathrm{ArH}^{+}\right)$and is obtained for an accumulation time of $50 \mathrm{~min}$ by using an $\mathrm{Ar}$ gas cell pressure of 2.15 
$\times 10^{-4}$ Torr and by setting the dc potential of the long octopole $0.4 \mathrm{~V}$ lower than that of the short octopole. Here, the resolution of the QMS is lowered to maximize the transmission of $\mathrm{Ar}^{+}$and $\mathrm{ArH}^{+}$product ions, and thus both $\mathrm{Ar}^{+}$and $\mathrm{ArH}^{+}$are transmitted. As shown in the PFI-PESICO TOF spectrum of Fig. 9(b), product $\mathrm{Ar}^{+} / \mathrm{ArH}^{+}$ions are slow ions and are spread in a temporal region of $\approx 200 \mu \mathrm{s}$. A velocity inversion of the TOF spectrum demonstrates that the product ions are all produced with near-thermal velocities. Figure 9(c) shows the PFI-PESICO TOF spectrum for product $\mathrm{Ar}^{+} / \mathrm{ArH}^{+}$obtained by setting the dc potential of the long octopole at $-2 \mathrm{~V}$ below that of the short octopole, in order to extract slow product $\mathrm{Ar}^{+} / \mathrm{ArH}^{+}$ions from the gas cell. As a result, the TOF distribution for product $\mathrm{Ar}^{+} / \mathrm{ArH}^{+}$observed in Fig. 9(c) is peaked at shorter flight times with a narrower temporal range of $\approx 100 \mu \mathrm{s}$. The intensities for reactant $\mathrm{H}_{2}{ }^{+}\left(v^{+}=2, N^{+}=1\right)$ and product $\mathrm{Ar}^{+} / \mathrm{ArH}^{+}$are measured by their coincidence counts after normalizing with the respective number of PFI-PE triggers. The absolute total cross sections for $\mathrm{Ar}^{+} / \mathrm{ArH}^{+}$can then be calculated by the ratio of the product and reactant ion intensities, the neutral reactant gas density, and the length of the gas cell.

In order to determine the branching ratios for $\mathrm{Ar}^{+}$and $\mathrm{ArH}^{+}$, the resolution for the QMS was increased to resolve these ions and PFI-PESICO TOF spectra for these ions were recorded. As an example, we show in Figs. 10(a), 10(b), and 10(c) the PFI-PESICO TOF spectra recorded at $E_{\text {lab }}$ $=2.5 \mathrm{eV}$ for reactant $\mathrm{H}_{2}{ }^{+}\left(v^{+}=2, N^{+}=0\right)$, product $\mathrm{ArH}^{+}$ and product $\mathrm{Ar}^{+}$, respectively. This measurement gives a $\mathrm{Ar}^{+}: \mathrm{ArH}^{+}$ratio of 1.3, which is consistent with results of previous experiments, indicating that at $E_{\mathrm{lab}}=2.5 \mathrm{eV}$ the cross section for charge transfer $\mathrm{Ar}^{+}$is $\approx 40 \%$ higher than that for $\mathrm{ArH}^{+}{ }^{3}$ As expected, Figs. 10(b) and 10(c) reveal that the arrival times for product $\mathrm{ArH}^{+}$ions are in the range of $\approx 100-200 \mu$ s, which is shorter than that of $\approx 100-250 \mu \mathrm{s}$ for charge transfer $\mathrm{Ar}^{+}$.

Although the intensities for PFI-PE vibrational bands of $\mathrm{H}_{2}^{+}\left(v^{+}>4\right)$ are significantly lower than those for $v^{+} \leqslant 4$, we have still been able to record the PFI-PESICO spectra for $\mathrm{Ar}^{+} / \mathrm{ArH}^{+}$from $\mathrm{H}_{2}^{+}\left(v^{+}>4, N^{+}\right)+\mathrm{Ar}$. As an illustration, we show in Figs. 11(a) and 11(b), the PFI-PESICO TOF spectra at $E_{\mathrm{lab}}=1.26 \mathrm{eV}$ for $\mathrm{H}_{2}{ }^{+}\left(v^{+}=6, N^{+}=1\right)$ and $\mathrm{Ar}^{+} / \mathrm{ArH}^{+}$. Absolute total cross sections for $\mathrm{Ar}^{+} / \mathrm{ArH}^{+}$ formed by the reaction $\mathrm{H}_{2}{ }^{+}\left(v^{+}, N^{+}=1\right)+$ Ar with $v^{+}=17$ have been reported recently. ${ }^{23}$

\section{ION TRAJECTORY CALCULATIONS}

In order to understand and to optimize the application of the ion gate, we have performed ion trajectory calculations between the PI/PEX region and the short rf octopole using the SIMION program. ${ }^{31}$ The ion lens system of interest is divided into finite sections. Based on the two-dimensional (or three-dimensional) geometries used, the SIMION program calculates the electrostatic potentials for the ion lens sections. Since individual sections can be modeled independently, higher resolution arrays can be used in more critical areas such as the wired ion gate lens. SIMION has the function to assemble individual potential arrays for modeling of

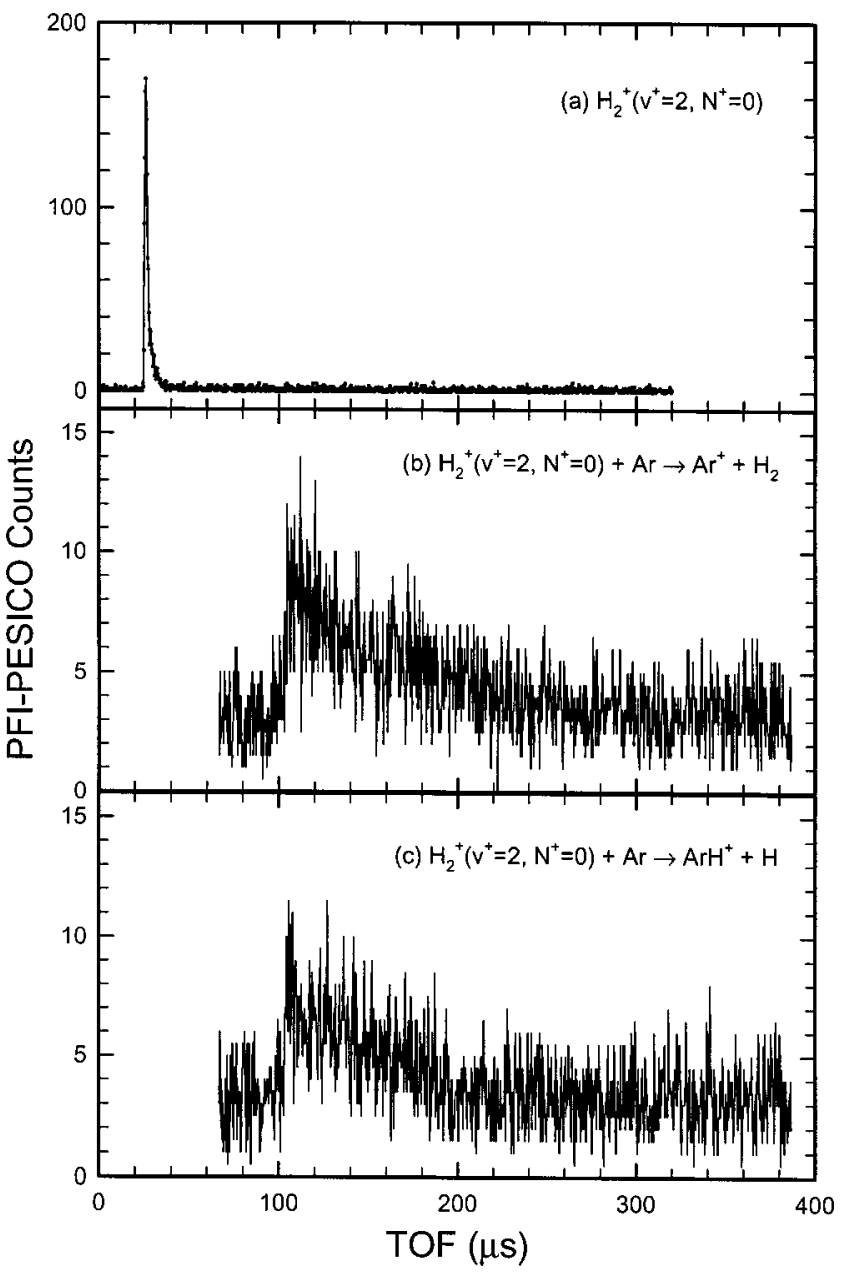

FIG. 10. PFI-PESICO TOF spectra for (a) $\mathrm{H}_{2}{ }^{+}\left(v^{+}=2, N^{+}=0\right)$, (b) $\mathrm{Ar}^{+}$ and (c) $\mathrm{ArH}^{+}$formed by the reaction of $\mathrm{H}_{2}{ }^{+}\left(v^{+}=2, N^{+}=0\right)+\mathrm{Ar}$ at $E_{\text {lab }}$ $=2.5 \mathrm{eV}$. The accumulation time used for each of the $\mathrm{Ar}^{+}$and $\mathrm{ArH}^{+}$spectra was $90 \mathrm{~min}$. The dc potential of the long octopole was set $-2 \mathrm{~V}$ lower than that of the short octopole.

the entire ion lens system. The trajectories of charged particles are calculated based on the modeled potential arrays of the entire ion lens system. We have also integrated the trajectory calculations to include rf fields applied to the octopoles and QMS. However, only the calculations involving the dc fields applied to the aperture lenses in the region between the photoionization region (9) and the short rf octopole that are relevant to understanding the performance of the wired ion gate lens are shown here.

In the simulation, ions were randomly generated inside a cubic volume of $0.3 \times 0.3 \times 0.3 \mathrm{~mm}^{3}$ centered at the photoionization center. This cubic volume is an estimate of the interaction volume of the VUV beam and the neutral gas sample. We note that this volume ignores ion and electrons generated along the VUV beam, particularly when the neutral precursor sample is introduced as an effusive beam. For ions produced with non-zero initial kinetic energies, velocity vectors are generated isotropically with a chosen vector origin situated inside the cubic interaction volume. The isotropic vector generation is ensured by the uniform vector density on the surface of a given vector sphere. The vector origins in the cubic photoionization volume are chosen ran- 


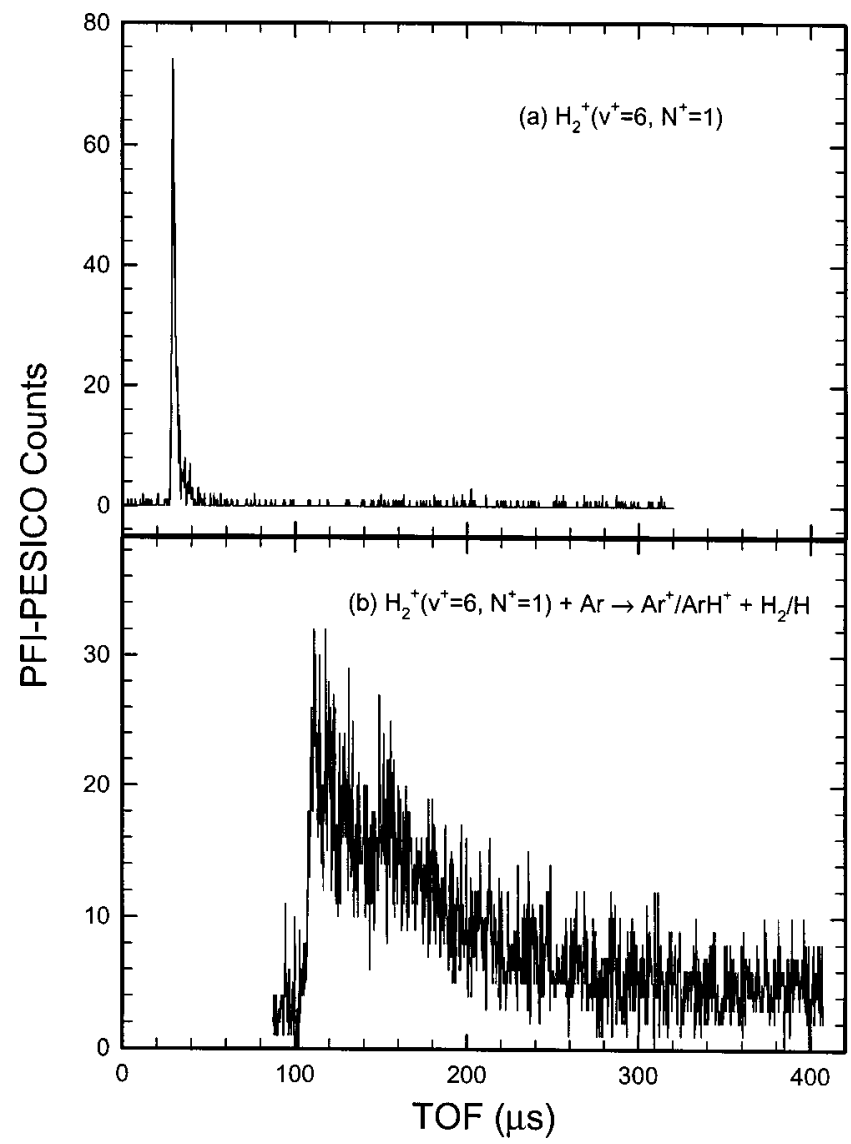

FIG. 11. PFI-PESICO TOF spectrum for (a) $\mathrm{H}_{2}{ }^{+}\left(v^{+}=6, N^{+}=1\right)$ and (b) $\mathrm{Ar}^{+} / \mathrm{ArH}^{+}$formed by the reaction of $\mathrm{H}_{2}^{+}\left(v^{+}=6, N^{+}=1\right)+\mathrm{Ar}$ at $E_{\mathrm{lab}}$ $=1.26 \mathrm{eV}$. The spectrum shown in (b) was obtained by setting the dc potential of the long octopole $-2 \mathrm{~V}$ lower than that of the short octopole. Accumulation time $=60 \mathrm{~min}$.

domly, reflecting on a uniform density for the neutral gas sample in the interaction volume.

\section{A. Ion TOF distribution observed at ion repeller plate}

When a dc field $[E(\mathrm{~V} / \mathrm{cm})]$ is maintained at the photoionization region, the ion TOF distribution observed at the grid of the ion repeller plate I1 (see Fig. 2) can be derived analytically. Let $D$ be the distance between the photoionization center and the grid of the ion repeller plate I1, $v$ equal to the initial ion velocity, $\theta$ equal to the angle between $v$ and the TOF axis, $m$ equal to the mass of the ion, $q$ equal to the electric charge of the ion, $\mathrm{KE}=(1 / 2) \mathrm{m} v^{2}$ equal to the initial ion kinetic energy, and $t$ equal to the time for an ion to exit the grid of the ion repeller I1, we can show that

$$
D=\left(\sqrt{\frac{2 \mathrm{KE}}{m}} \cos \theta\right) t+\frac{q E}{2 m} t^{2} .
$$

Based on Eq. (4), we have

$$
t=\frac{\sqrt{2 m}}{q E}\left(\sqrt{\mathrm{KE} \cos ^{2} \theta+q E D}-\sqrt{\mathrm{KE}} \cos \theta\right) .
$$

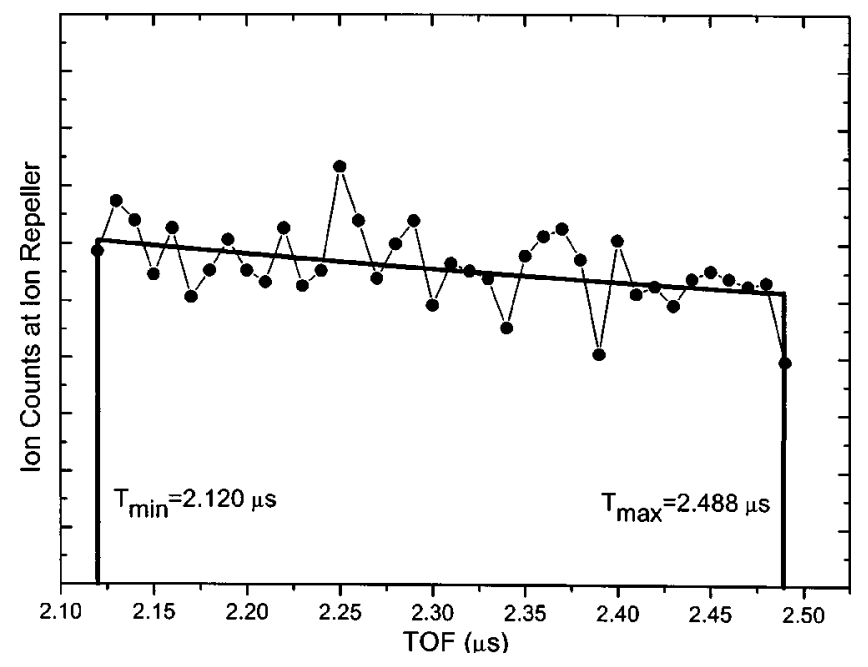

FIG. 12. TOF spectra for $\mathrm{Ar}^{+}$formed by photoionization of an effusive $\mathrm{Ar}$ sample with a kinetic energy $\mathrm{KE}=26 \mathrm{meV}$. (solid line): predicted by Eqs. (11) and (12) and (solid dots): obtained by ion trajectory calculations based on 3000 trajectories using the SIMION program. $T_{\min }$ and $T_{\max }$ are the minimum and maximum times predicted by Eqs. (10a) and (10b).

$$
\cos \theta=\frac{2 m D-q E t^{2}}{2 t \sqrt{2 \mathrm{KE} m}} .
$$

For a given $\theta$, the vector sphere surface element is $d S$ $=2 \pi v^{2} \sin \theta d \theta$. Thus, the intensity $d I$ observed due to ions with velocity vectors ending on the surface element $d S$ is proportional to $d S$ :

$$
d I=\rho d S=2 \pi v^{2} \rho \sin \theta d \theta=\frac{4 \pi K E \rho}{m} \sin \theta d \theta .
$$

Here, $\rho$ is the surface density of velocity vectors and is a constant for an isotropic distribution. From Eq. (6), we obtain

$$
d(\cos \theta)=\frac{-1}{\sqrt{2 \mathrm{KE} m}}\left[\frac{m D}{t^{2}}+\frac{1}{2} q E\right] d t .
$$

Combining Eqs. (7) and (8), we obtain the TOF distribution as

$$
\frac{d I}{d t}=\frac{2 \pi \rho}{m} \sqrt{\frac{2 \mathrm{KE}}{m}}\left[\frac{m D}{t^{2}}+\frac{1}{2} q E\right] .
$$

Equation (5) gives the minimum and maximum values of $t$ :

$$
\begin{aligned}
& t_{\min }=\frac{\sqrt{2 m}}{q E}(\sqrt{\mathrm{KE}+q E D}-\sqrt{\mathrm{KE}}) \\
& t_{\max }=\frac{\sqrt{2 m}}{q E}(\sqrt{\mathrm{KE}+q E D}+\sqrt{\mathrm{KE}}) .
\end{aligned}
$$

Figure 12 depicts the TOF distribution for $\mathrm{Ar}^{+}$(solid line) formed by photoionization of a sample with a single kinetic energy of $26 \mathrm{meV}$ as predicted by Eqs. (9) and (10) for $D=0.50 \mathrm{~cm}$ and $E=8 \mathrm{~V} / \mathrm{cm}(-4.0$ at repeller I1 and 


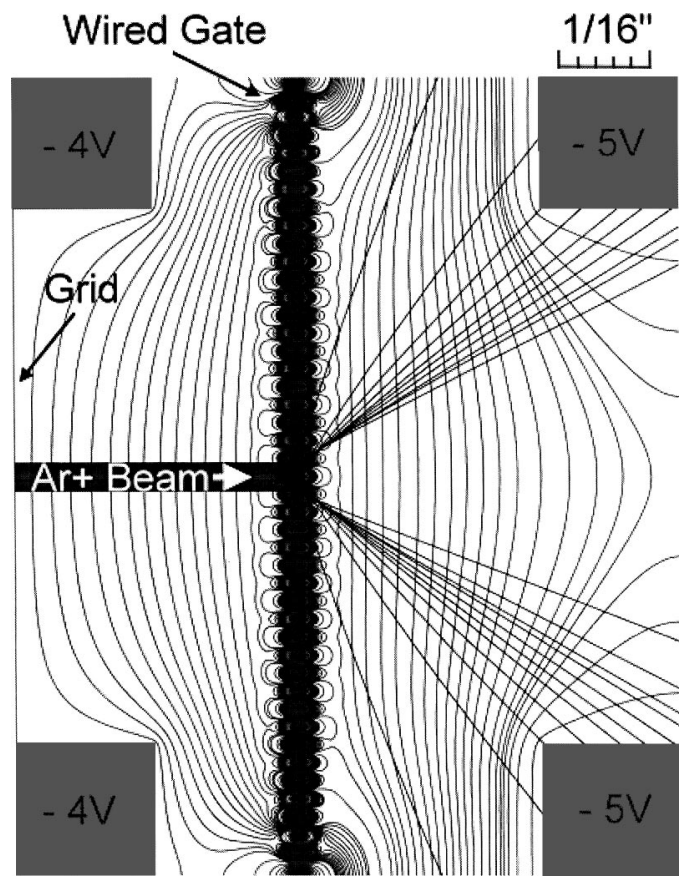

FIG. 13. Ion trajectories deflected by the wired ion gate lens and equipotential lines in the vicinity of the wired ion gate lens. The wired ion gate lens was floated at a de potential of $-4.4 \mathrm{~V}$. On top of this de field, potentials of +4.4 and $-4.4 \mathrm{~V}$ were applied to the even and odd sets of wires, respectively. That is, net potentials for even wires were zero, while that for odd wires were $-8.8 \mathrm{~V}$. The dc potentials applied to I1 and the adjacent lens on the right are -4.0 and $-5.0 \mathrm{~V}$, respectively.

$+4.0 \mathrm{~V}$ at repeller E1). This single kinetic energy calculation is a model for a thermal gas source in which the average kinetic energy is $26 \mathrm{meV}$. The $\rho$ value can be viewed as a scaling parameter. We have also obtained the TOF spectrum for $\mathrm{Ar}^{+}$(solid dots) based on ion trajectory calculations. Since only 3000 trajectories are sampled, the fluctuation observed in the latter spectrum is statistical in nature. As shown in Fig. 12, the TOF spectra for $\mathrm{Ar}^{+}$obtained by trajectory sampling and by Eqs. (9) and (10) are in excellent agreement. This observation can be taken as a test of the ion trajectory calculations, validating the calculation procedures based on the SIMION program.

\section{B. Calculations of ion trajectories and TOF spectra}

Figure 13 depicts the ion trajectories calculated using the SIMION program as they pass through the ion gate with the ion gate off, i.e., deflection fields on. As shown by the electrostatic equipotential lines shown in Fig. 13, strong local deflection fields exist between adjacent wires of the wired ion gate lens. With the exception of these short-range local fields around the wires, the equi-potential lines of Fig. 13 are essentially identical to those observed when the deflection fields between adjacent wires were turned off. The simulation shows that the deflecting fields between adjacent wires resulting from the positive and negative potentials applied to the even and odd sets of wires do not affect the ion trajectories at a distance of $<1 \mathrm{~mm}(\approx 3$ times the wire spacing $)$ away from the ion gate.

The analytical solution for the ion TOF spectrum observed in the PFI experiment is complicated by the pulsed

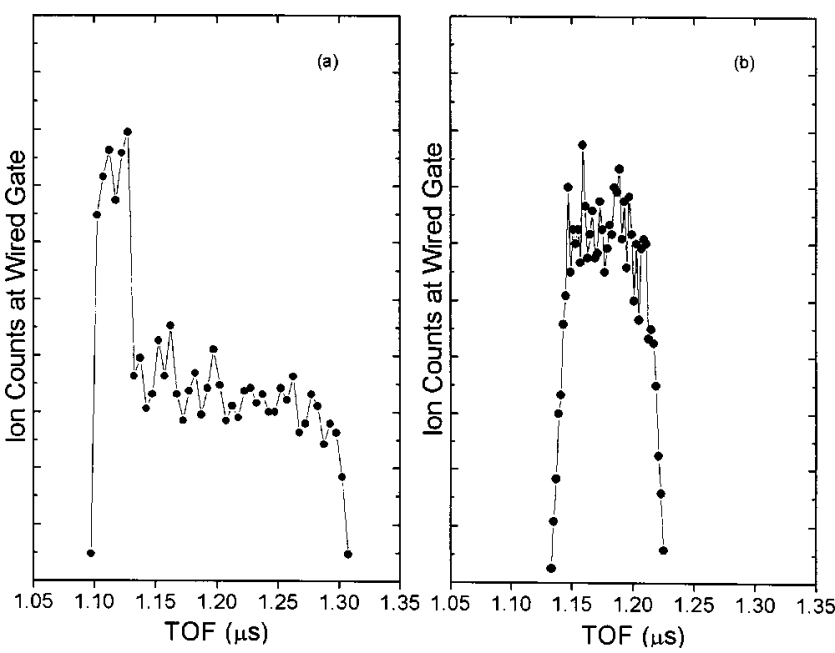

FIG. 14. TOF spectrum for PFI $\mathrm{H}_{2}{ }^{+}$ions (dots) formed by PFI of a VUV excited effusive $\mathrm{H}_{2}$ sample ( $\mathrm{KE}=26 \mathrm{meV}$ ) as observed at the wired ion gate lens. Time zero corresponds to the beginning of the ALS dark gap. The PFI-PIs were formed at $10 \mathrm{~ns}$ when the PFI field was switched on. (b) TOF spectrum for PFI $\mathrm{H}_{2}{ }^{+}$formed by the PFI of a VUV excited supersonic beam sample (KE of $3 \mathrm{meV}$ ). Three thousand trajectories were calculated for PFI-PIs.

nature of the ion extraction field. However, the ion TOF spectrum resulting from the PFI ion extraction can be estimated by ion trajectory calculations. The calculated ion TOF spectrum would yield estimates for the delay and gate width for turning on the wired ion gate to pass the PFI-PI. Figure 14(a) shows the TOF spectrum for PFI $\mathrm{H}_{2}{ }^{+}$ions formed by PFI of high- $n(n \geqslant 100)$ Rydberg $\mathrm{H}_{2}$ observed at the wired ion gate lens. Since the $\mathrm{H}_{2}$ sample was introduced as an effusive beam, we have assumed that the average kinetic energy for $\mathrm{H}_{2}{ }^{+}$was $26 \mathrm{meV}$. In this calculation, time zero is taken to be the beginning of the dark gap. We assume that the PFI-PIs were formed immediately as the PFI field was turned on (10 ns with respect to the beginning of the dark gap).

As shown in Fig. 14(a), PFI $\mathrm{H}_{2}{ }^{+}$ions are predicted to arrive at the ion gate between 1.10 and $1.31 \mu \mathrm{s}$. Thus, in the state selection of $\mathrm{H}_{2}{ }^{+}$using the PFI-PEPICO scheme, the delay and the gate width for opening the ion gate should be set at $1.1 \mu \mathrm{s}$ and $210 \mathrm{~ns}$, respectively. These values are consistent with the delay $(\approx 1.2 \mu \mathrm{s})$ and gate width $(250 \mathrm{~ns})$ used in the experimental studies of the state-selected reactions $\mathrm{H}_{2}{ }^{+}\left(v^{+}, N^{+}\right)+\mathrm{Ar}$. We find that the shape of the TOF spectrum depends sensitively on the initial ion KE and the position where the PFI-PIs are born in the photoionization region. The strong peak observed at $1.10-1.14 \mu \mathrm{s}$ in the TOF spectrum of Fig. 14(a) results from the pulse nature of the PFI field used for ion extraction.

Figures 15(a) and 15(b) depict the trajectories for PFI $\mathrm{H}_{2}{ }^{+}$ions and prompt background $\mathrm{H}_{2}{ }^{+}$ions, respectively, between the photoionization center and the entrance of the short rf-octopole ion guide. Employing the delay of $1.1 \mu \mathrm{s}$ and gate width of $250 \mathrm{~ns}$ for turning on the wired ion gate, the simulation of Fig. 15(a) indicates that $96 \%$ of the PFI $\mathrm{H}_{2}{ }^{+}$ions pass the ion gate and are focused by the ion injection lenses into the short rf-octopole ion guide. For prompt background $\mathrm{H}_{2}{ }^{+}$ions, which arrive at the ion gate when the ion gate is "off," the trajectory calculation of Fig. 15(b) 

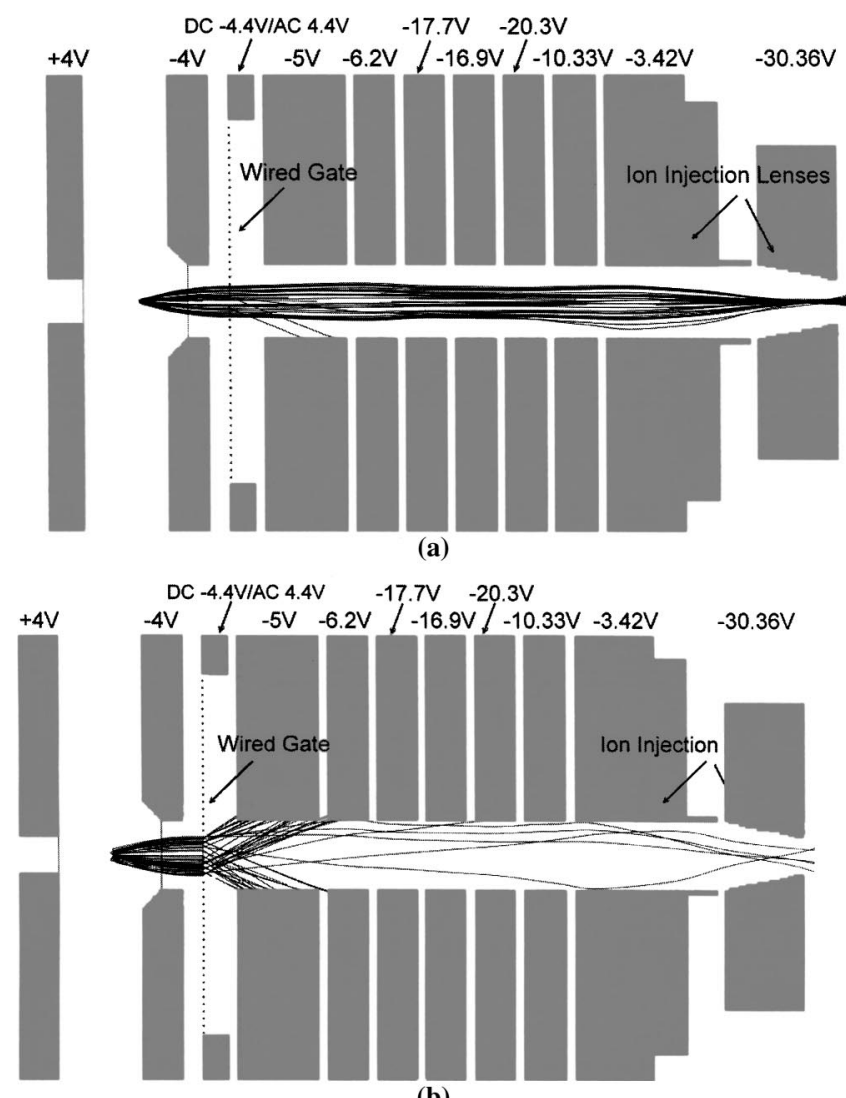

(b)

FIG. 15. (a) Trajectories for PFI $\mathrm{H}_{2}{ }^{+}$ions between the PI/PEX region and the short rf octopole. The wired ion gate (gate width $=250 \mathrm{~ns}$ ) was turned on at a delay of $1.1 \mu \mathrm{s}$ with respect to the formation of the PFI $\mathrm{H}_{2}{ }^{+}$. The trajectory calculations show that $96 \%$ of the PFI-PIs were transmitted. (b) Trajectories for prompt background $\mathrm{H}_{2}{ }^{+}$ions between the PI/PEX region and the short octopole. The prompt ions arrived at the wired ion gate lens when the wired ion gate is turned "off." The trajectory calculations show that $96 \%$ of the ions were rejected.

shows that $96 \%$ of the prompt background $\mathrm{H}_{2}{ }^{+}$ions are rejected by the ion gate. The calculation also shows that the ion injection lenses [see (12) of Figs. 1 and 2] are important elements for efficient injection of reactant ions into the short rf octopole at different $E_{\text {lab }}$ values.

Figure 16(a) shows the calculated TOF spectrum for $\mathrm{Ar}^{+}$ PFI ions from the photoionization region to the ion gate. The calculations assume that the PFI $\mathrm{Ar}^{+}$ions are formed from an Ar sample with a KE of $26 \mathrm{meV}$. The $\mathrm{Ar}^{+} \mathrm{TOF}$ distribution of Fig. 16(a) exhibits two prominent peaks at 5.35 and $6.05 \mu$ s. Similar to the TOF spectrum for PFI $\mathrm{H}_{2}{ }^{+}$, the trajectory calculations show that these peaks result from the pulse nature of the PFI ion extraction field. The full width of the $\mathrm{Ar}^{+}$spectrum indicates that the use of an ion gate width of $\approx 1.1 \mu$ s is required to transmit all PFI $\mathrm{Ar}^{+}$through the wired ion gate lens.

The ion gate width of $200 \mathrm{~ns}$ used in recording the PFIPEPICO spectra of Figs. 5(b), 5(c), and 6(a)-6(c) was obtained by maximizing the coincidence ion counts, i.e., the difference in ion counts between the first ion gate and the second ion gate. These measurements were made prior to the ion trajectory calculations. Obviously, the $200 \mathrm{~ns}$ ion gate width used is significantly narrower than the predicted full width of $1.1 \mu \mathrm{s}$, and is thus expected to reduce the $\mathrm{Ar}^{+}$ion
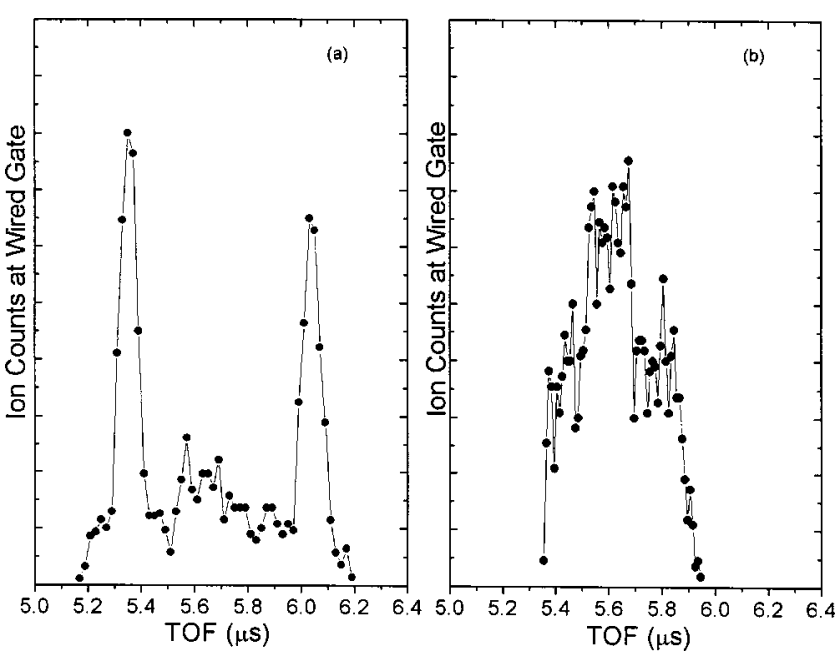

FIG. 16. Calculated TOF spectra for $\mathrm{Ar}^{+}$formed by PFI of a VUV excited (a) effusive Ar sample with a KE of $26 \mathrm{meV}$. (b) Supersonic Ar beam with a KE of $3 \mathrm{meV}$.

transmission in the PFI-PEPICO measurement. The calculated TOF spectrum for $\mathrm{Ar}^{+}$shown in Fig. 16(a) suggests that the ion gate of $200 \mathrm{~ns}$ in the PFI-PEPICO measurements (Figs. 5 and 6) was most likely centered at the strong TOF peak for $\mathrm{Ar}^{+}$at $5.35 \mu \mathrm{s}$. In the PFI-PEPICO measurements of $\operatorname{Ar}^{+}\left({ }^{2} \mathrm{P}_{3 / 2,1 / 2}\right)$, the actual delay (with respect to the PFI-PE trigger pulse) used for turning off the deflection field of the wired ion gate is $4.7 \mu \mathrm{s}$. The difference in the calculated and experimental delays can be attributed to the electron flight time and a finite delay due to the electronics. In any case, the calculated TOF distribution shown in Fig. 16(a) serves to provide a rationalization for the use of a $200 \mathrm{~ns}$ ion gate width in the PFI-PEPICO measurements shown in Figs. 5 and 6.

\section{POSSIBLE IMPROVEMENTS OF PFI-PEPICO AND PFI-PESICO MEASUREMENTS}

The preparation of state-selected reactant ions using the PFI-PEPICO method along with the wired ion gate scheme, can be significantly improved if the neutral gas sample is introduced to the PI/PEX region in the form of a supersonic molecular beam traveling in a direction perpendicular to the ion TOF axis or the axis of the octopole-quadrupole assembly. We note that the rotational cooling achieved by the supersonic beam method is essential in some experiments for obtaining a better-resolved PFI-PE spectrum for the reactant ion of interest. As shown in the analysis below, due to the low translational temperature achieved in the supersonic beam arrangement, the FWHM of the coincidence ion TOF peak is significantly reduced as compared to that observed in using an effusive beam sample. Furthermore, the supersonic beam method also can reduce the kinetic energy spread for the reactant ions in PFI-PESICO measurements.

Figure 14(b) depicts the calculated TOF spectrum for PFI $\mathrm{H}_{2}{ }^{+}$ions formed by PFI of a VUV excited $\mathrm{H}_{2}{ }^{+}$sample with a translational temperature of 20-30 K (or an average $\mathrm{KE}$ of $3 \mathrm{meV}$ ) along the ion TOF axis. As expected, the full width (95 ns) of the TOF distribution observed in Fig. 14(b) is considerably narrower than that (210 ns) of Fig. 14(a). If a 

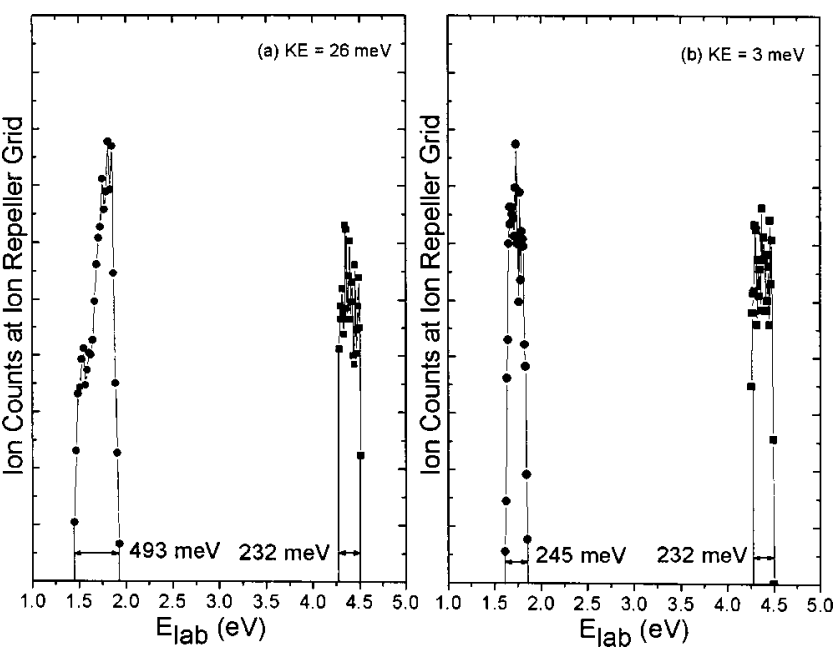

FIG. 17. Comparison of the calculated $E_{\text {lab }}$ distribution for $\mathrm{H}_{2}{ }^{+}$(observed at the grid of the ion repeller I1) extracted using the PFI ion extraction scheme with that using the dc ion extraction arrangement. (a) $\mathrm{The}_{2}{ }^{+}$ions are assumed to be formed by photoionization of an effusive $\mathrm{H}_{2}$ sample with a $\mathrm{KE}$ of $26 \mathrm{meV}$. The calculation gives $E_{\mathrm{lab}}=4.25 \mathrm{eV}$ and $\Delta E_{\mathrm{lab}}$ $=0.232 \mathrm{eV}$ for the $\mathrm{dc}$ ion extraction arrangement, whereas the calculation predicts $E_{\mathrm{lab}} \approx 1.75 \mathrm{eV}$ and $\Delta E_{\mathrm{lab}}=0.493 \mathrm{eV}$ for the PFI ion extraction mode. (b) The $\mathrm{H}_{2}{ }^{+}$ions are assumed to be formed by photoionization of a supersonically cooled $\mathrm{H}_{2}$ sample with a $\mathrm{KE}$ of $3 \mathrm{meV}$. The calculation predicts $E_{\mathrm{lab}} \approx 4.25 \mathrm{eV}$ and $\Delta E_{\mathrm{lab}}=0.232 \mathrm{eV}$ for the dc ion extraction arrangement and $E_{\mathrm{lab}} \approx 1.75 \mathrm{eV}$ and a $\Delta E_{\mathrm{lab}}=0.245 \mathrm{eV}$ for the PFI ion extraction scheme.

$\mathrm{H}_{2}$ supersonic beam of $20-30 \mathrm{~K}$ is used to introduce the $\mathrm{H}_{2}$ sample into the PI/PEX center, the wired ion gate width can be reduced to $95 \mathrm{~ns}$, which should result in a reduction factor of $0.95 \times 10^{-7}$ for background false coincidences as compared to that $\left(2.1 \times 10^{-7}\right)$ expected in using the effusive beam. For an $\mathrm{Ar}^{+}$kinetic energy of $3 \mathrm{meV}$ (or translational temperature of 20-30 K), the $\mathrm{Ar}^{+} \mathrm{TOF}$ distribution has a full width of $\approx 550 \mathrm{~ns}$ and is peaked at $5.6 \mu$ s [see Fig. 16(b)]. That is, an ion gate width of $\approx 550 \mathrm{~ns}$ can be used to transmit all $\mathrm{Ar}^{+}$PFI ions formed from a supersonically cooled $\mathrm{Ar}$ sample at $20-30 \mathrm{~K}$. To transmit all $\mathrm{Ar}^{+}$PFI-PIs in the present experiment would require a gate width of $1100 \mathrm{~ns}$ [see Fig. 16(a)]. We note that the achievement of a narrow PFI-PEPICO TOF distribution for the reactant ions would also allow the use of the wired ion gate as a mass filter to reject background ions originating from the photoionization region with masses different from that of the reactant ion of interest.

The trajectory calculations also reveal that the pulse nature of the PFI ion extraction field has a strong effect on the achievable laboratory kinetic energy resolution $\Delta E_{\text {lab }}$ for the reactant ions. Figure 17(a) compares the $E_{\text {lab }}$ distribution for $\mathrm{H}_{2}{ }^{+}$extracted using the PFI pulsed ion extraction scheme as described above with that using dc voltages of $+4.0 \mathrm{~V}$ at the electron repeller plate $\mathrm{E} 1$ and $-4.0 \mathrm{~V}$ at the ion repeller plate I1. In this calculation, we assume that the $\mathrm{H}_{2}{ }^{+}$ions are formed by photoionization of an effusive $\mathrm{H}_{2}$ sample with a $\mathrm{KE}$ of $26 \mathrm{meV}$. For the dc ion extraction arrangement, the calculation gives $E_{\mathrm{lab}} \approx 4.25 \mathrm{eV}$ and $\Delta E_{\mathrm{lab}}=0.232 \mathrm{eV}$ as observed at the grid of the ion repeller plate I1 (see Fig. 2), whereas for the PFI ion extraction mode, we obtain $E_{\text {lab }}$ $\approx 1.75 \mathrm{eV}$ and $\Delta E_{\text {lab }}=0.493 \mathrm{eV}$. The lower $E_{\text {lab }}$ observed for the PFI ion extraction mode results from the shorter acceleration time for the reactant ions. These predicted $\Delta E_{\text {lab }}$ values are consistent with the measured $\Delta E_{\text {lab }}$ values using the retarding potential method. Assuming that the $\mathrm{H}_{2}{ }^{+}$ions are formed by photoionization of a supersonically cooled $\mathrm{H}_{2}$ sample at $20-30 \mathrm{~K}$ or a $\mathrm{KE}$ of $3 \mathrm{meV}$, we have calculated the $E_{\text {lab }}$ distributions for $\mathrm{H}_{2}{ }^{+}$using the PFI ion extraction scheme and the dc ion extraction arrangement [see Fig. 17(b) ]. As shown in Figs. 17(a) and 17(b), the $\Delta E_{\text {lab }}$ values $(0.232 \mathrm{eV})$ for $\mathrm{H}_{2}{ }^{+}$formed by dc ion extraction of effusive and supersonic beam samples are predicted to be essentially identical. However, the $\Delta E_{\mathrm{lab}}=0.245 \mathrm{eV}$ for $\mathrm{H}_{2}{ }^{+}$formed by PFI of a supersonically cooled $\mathrm{H}_{2}$ sample is found to be a factor of 2 less than the value of $\Delta E_{\mathrm{lab}}=0.493 \mathrm{eV}$ for $\mathrm{H}_{2}{ }^{+}$ produced by PFI of an effusive $\mathrm{H}_{2}$ sample.

\section{ACKNOWLEDGMENTS}

This work has been supported by AFOSR through Task No. 2303EP02 and Grant No. F49620-99-1-0234 (Program Manager: Michael R. Berman). One of the authors (C.Y.N.) also acknowledges support by NSF Grant No. ATM-0001646 and partial support by the U.S. Department of Energy, Office of Basic Energy Sciences, Division of Chemical Sciences, Geosciences, and Biosciences. One of the authors (J.S.M.) was the beneficiary of a NRC postdoctoral fellowship.

${ }^{1}$ W. A. Chupka, in Ion-Molecule Reactions, edited by J. L. Franklin (Plenum, New York, 1972), p. 33.

${ }^{2}$ C. Y. Ng, in Techniques for the Study of Gas-Phase Ion-Molecule Reactions, edited by J. M. Farrar and W. H. Saunder, Jr. (Wiley, New York, 1988), p. 417.

${ }^{3}$ C. Y. Ng, Adv. Chem. Phys. 82, 401 (1992).

${ }^{4}$ C. Y. Ng, J. Phys. Chem. A 106, 5952 (2002).

${ }^{5}$ I. Koyano and K. Tanaka, Adv. Chem. Phys. 82, 263 (1992).

${ }^{6}$ O. Dutuit, C. Alcaraz, D. Gerlich, P. M. Guyon, J. Hepburn, C. MetayerZeitoun, J. B. Ozenne, M. Schweizer, and T. Weng, Chem. Phys. 209, 177 (1996).

${ }^{7}$ S. L. Anderson, Adv. Chem. Phys. 82, 213 (1992).

${ }^{8}$ T. Baer and P. M. Guyon, in High Resolution Laser Photoionization and Photoelectron Studies, edited by I. Powis, T. Baer, and C. Y. Ng, Wiley Series in Ion Chemistry and Physics (Wiley, Chichester, 1995), p. 1.

${ }^{9}$ R. I. Hall, A. McConkey, K. Ellis, G. Dawber, L. Avaldi, M. A. MacDonald, and G. C. King, Meas. Sci. Technol. 3, 316 (1992).

${ }^{10}$ Y. Morioka, Adv. Ser. Phys. Chem. 10A, 347 (2000).

${ }^{11}$ K. Müller-Dethlefs, M. Sander, and E. W. Schlag, Chem. Phys. Lett. 112, 291 (1984).

${ }^{12}$ E. W. Schlag, ZEKE Spectroscopy (Cambridge University Press, Cambridge, U.K., 1996).

${ }^{13}$ P. M. Johnson, Adv. Ser. Phys. Chem. 10A, 296 (2000).

${ }^{14}$ C. Y. Ng, Annu. Rev. Phys. Chem. 53, 101 (2002).

${ }^{15}$ C. Y. Ng, Adv. Ser. Phys. Chem. 10, 394 (2000).

${ }^{16}$ C. Y. Ng, Int. J. Mass. Spectrom. 200, 357 (2000).

${ }^{17}$ G. K. Jarvis, K.-M. Weitzel, M. Malow, T. Baer, Y. Song, and C. Y. Ng, Rev. Sci. Instrum. 70, 3892 (1999).

${ }^{18}$ P. Heimann, M. Koike, C.-W. Hsu, D. Blank, X. M. Yang, A. Suits, Y. T. Lee, M. Evans, C. Y. Ng, C. Flaim, and H. A. Padmore, Rev. Sci. Instrum. 68, 1945 (1997).

${ }^{19}$ N. E. Bradbury and R. A. Nielsen, Phys. Rev. 49, 388 (1936).

${ }^{20}$ P. R. Vlasak, D. J. Beussman, M. R. Davenport, and C. G. Enke, Rev. Sci. Instrum. 67, 68 (1996).

${ }^{21}$ K. Norwood and C. Y. Ng, Chem. Phys. Lett. 156, 145 (1989).

${ }^{22}$ C. Y. Ng, in Vacuum Ultraviolet Photoionization and Photodissociation of Molecules and Clusters, edited by C. Y. Ng (World Scientific, Singapore, 1991), p. 169. 
${ }^{23}$ X. M. Qian, T. Zhang, Y. Chiu, D. J. Levandier, J. S. Miller, R. A. Dressler, and C. Y. Ng, J. Chem. Phys. 118, 2455 (2003).

${ }^{24}$ D. Gerlich, Adv. Chem. Phys. 82, 1 (1992).

${ }^{25}$ N. R. Daly, Rev. Sci. Instrum. 31, 264 (1960).

${ }^{26}$ R. A. Dressler (unpublished).

${ }^{27}$ K. M. Ervin and P. B. Armentrout, J. Chem. Phys. 83, 166 (1985).
${ }^{28}$ R. A. Dressler, Y.-H. C. Chiu, D. J. Levandier, and C. Y. Ng, J. Chem. Phys. 113, 8561 (2000).

${ }^{29}$ S. Stimson, Ph.D. thesis, Iowa State University (1998)

${ }^{30}$ S. Stimson, Y.-J. Chen, M. Evans, C.-L. Liao, C. Y. Ng, C.-W. Hsu, and P. Heimann, Chem. Phys. Lett. 289, 507 (1998).

${ }^{31}$ D. A. Dahl, in Proceedings of the 43rd ASMS Conference on Mass Spectrometry and Allied Topics, Atlanta, GA, 21-26 May (1995), p. 717. 